\title{
Convergence of derivative expansion in supersymmetric functional RG flows
}

\author{
Marianne Heilmann, Tobias Hellwig, Benjamin Knorr, Marcus Ansorg and \\ Andreas Wipf \\ Theoretisch-Physikalisches Institut, Friedrich-Schiller-Universität Jena, \\ Max-Wien-Platz 1, D-07743 Jena, Germany \\ E-mail: marianne.heilmann@uni-jena.de, tobias.hellwig@uni-jena.de, \\ benjamin.knorr@uni-jena.de, marcus.ansorg@uni-jena.de, \\ wipf@tpi.uni-jena.de
}

ABSTRACT: We confirm the convergence of the derivative expansion in two supersymmetric models via the functional renormalization group method. Using pseudo-spectral methods, high-accuracy results for the lowest energies in supersymmetric quantum mechanics and a detailed description of the supersymmetric analogue of the Wilson-Fisher fixed point of the three-dimensional Wess-Zumino model are obtained. The superscaling relation proposed earlier, relating the relevant critical exponent to the anomalous dimension, is shown to be valid to all orders in the supercovariant derivative expansion and for all $d \geq 2$.

KEYwords: Superspaces, Supersymmetric Effective Theories, Renormalization Group, Nonperturbative Effects

ARXIV EPRINT: 1409.5650 


\section{Contents}

1 Introduction 1

2 Supersymmetric quantum mechanics $\quad 3$

2.1 Flow equation in superspace 4

2.1.1 Supercovariant derivative expansion in NNLO 5

2.1.2 Introducing the regulator functional 5

$\begin{array}{lll}2.1 .3 & \text { Flow equation } & 6\end{array}$

$\begin{array}{lll}2.2 & \text { Effective potential and first excited energy } & 7\end{array}$

2.2.1 On-shell effective potential $\quad 8$

$\begin{array}{lll}2.2 .2 & \text { Numerical results } & 9\end{array}$

$\begin{array}{lll}2.3 & \text { Supersymmetry breaking } & 12\end{array}$

2.3.1 Problems with the expansion in powers of $F \quad 13$

$\begin{array}{lll}2.3 .2 & \text { Numerical results } & 14\end{array}$

$3 \mathcal{N}=1$ Wess-Zumino model in 3 dimensions $\quad 15$

$\begin{array}{llr}3.1 & \text { Preliminaries } & 15\end{array}$

$\begin{array}{ll}3.2 \text { Derivation of flow equation } & 16\end{array}$

$\begin{array}{lll}3.3 & \text { Superscaling relation \& Wilson-Fisher fixed point } & 17\end{array}$

$\begin{array}{lll}4 & \text { Summary } & 20\end{array}$

$\begin{array}{ll}\text { A Greens function in } d=1 & 21\end{array}$

B Flow equation of $Y_{1}^{\prime}+Y_{2}$

$\begin{array}{ll}\text { C Greens function in } d=3 & 23\end{array}$

D (Pseudo-)spectral methods $\quad 24$

\section{Introduction}

Supersymmetry (SUSY) is an essential ingredient in most theories beyond the Standard Model of particle physics. Under certain natural conditions it is the unique extension of the Poincaré symmetry. Although many predictions of supersymmetry can be explored by perturbative calculations there remain interesting phenomena which fall into the nonperturbative regime. Examples are collective condensation phenomena, topological effects and supersymmetry breaking. Thus, powerful methods are needed to investigate and calculate such non-perturbative effects in order to understand the underlying physics and to obtain quantitative predictions in the strong-coupling regime. 
A well-established method to study non-perturbative effects in supersymmetric theories is based on a discretization of spacetime and the corresponding supersymmetric lattice models, see e.g. [1-8]. Yet, as supersymmetry involves spacetime translations and rotations, lattice approaches usually go along with a complete (for models with extended supersymmetry partial) loss of supersymmetry.

In this work, we choose an alternative approach based on the functional renormalization group (FRG). The FRG is a powerful non-perturbative tool to study continuum physics and was successfully applied to a plethora of physical systems which include fermionic systems, gauge theories or gravity, see e.g. [9-14]. Only lately, the FRG has been applied to SUSY models - mainly due to the difficulty of constructing a sensible regularization that preserves SUSY. Here, we will extend previous results [15-17] by examining whether the derivative expansions for supersymmetric quantum mechanics or supersymmetric Yukawa theories are convergent, and further investigate SUSY breaking in these models. To that aim we admit higher order supercovariant derivative terms in the effective actions of supersymmetric Wess-Zumino-models in various dimensions.

In the early days of the FRG, ordinary quantum mechanics was already utilized as toy model to test the power of the techniques beyond perturbation theory. In (supersymmetric) quantum mechanics, flow equations are easily obtained and solutions to truncated flow equations can be compared with exact results. The focus in these studies were the energies of the low lying states [18-22]. It turned out that systems with single-well potentials can be successfully described all the way from weak to strong couplings, whereas double-wells are more challenging, partially due to exponentially suppressed instanton effects.

The cited works satisfactorily calculated the energy spectrum of non-supersymmetric systems. In non-supersymmetric systems there is no need to relate the regulators of bosons and fermions. However, in a supersymmetric systems they must be related by supersymmetry in order to disentangle the effects of spontaneous and explicit supersymmetry breaking. Thus in the present work we use manifestly supersymmetric regulators such that for supersymmetric initial conditions the scale dependent effective action is supersymmetric at all scales. This way we considerably improve upon previous results obtained in [15]. Other works using a supersymmetric regularization include [23-25].

Regarding the models investigated in the present work, we shall see that the supercovariant derivative expansion converges nicely. To calculate the flow of the superpotential and wave functions renormalizations to fourth order in this expansion with high precision we employ the powerful spectral method [26]. This method has been very successfully applied to problems in hydrodynamics, quantum chemistry and in particular gravity [27]. To solve the system of coupled nonlinear partial differential equations with spectral methods we use the Chebyshev polynomials as basis in the domain where the effective potential is flat or concave and rational Chebyshev functions in the domains where the effective potential is convex. For the RG-evolution, another Chebyshev spectralization was used. This way we are able to construct global solutions to the truncated flow equations with unmatched numerical accuracy.

The paper is organized as follows: in section 2 we review the relevant features of SUSY quantum mechanics. Sections 2.1 and 2.2 contain the derivation of the flow equations in 
next-to-next-to-leading order (NNLO) as well as numerical results for the first excited energies in systems with unbroken supersymmetry. Spontaneous supersymmetry breaking is analyzed in next-to-leading order (NLO) in section 2.3. In section 3 we proceed with the derivative expansion of the three-dimensional Wess-Zumino models. The truncated flow equations are analysed in section 3, which contains an analysis of the physics of the Wilson-Fisher fixed point and the superscaling relation previously discovered in [28]. The appendices contain further details on the derivation of the flow equations and on the spectral method used in the present work.

\section{Supersymmetric quantum mechanics}

In order to derive the flow equations for supersymmetric quantum mechanics, we employ the superfield formalism [29]. For more details we refer the reader to [15]. The Euclidean superfield, expanded in terms of the anticommuting Grassmann variables $\theta$ and $\bar{\theta}$, reads

$$
\Phi(\tau, \theta, \bar{\theta})=\phi(\tau)+\bar{\theta} \psi(\tau)+\bar{\psi} \theta(\tau)+\bar{\theta} \theta F(\tau) .
$$

Both the superfield and the Grassmann variables $\theta, \bar{\theta}$ have mass dimension $-1 / 2$. Next, we introduce the superpotential and expand it in powers of the Grassmann variables,

$$
W(\Phi)=W(\phi)+(\bar{\theta} \psi+\bar{\psi} \theta) W^{\prime}(\phi)+\bar{\theta} \theta\left(F W^{\prime}(\phi)-W^{\prime \prime}(\phi) \bar{\psi} \psi\right) .
$$

The one-dimensional equivalent of the Super-Poincaré algebra contains only translations of Euclidean time and is generated by one pair of conserved nilpotent fermionic supercharges $\mathcal{Q}=i \partial_{\bar{\theta}}+\theta \partial_{\tau}$ and $\overline{\mathcal{Q}}=i \partial_{\theta}+\bar{\theta} \partial_{\tau}$. The anticommutator of them is the super-Hamiltonian,

$$
\{\mathcal{Q}, \overline{\mathcal{Q}}\}=2 H, \quad[H, \mathcal{Q}]=[H, \overline{\mathcal{Q}}]=0 .
$$

Supersymmetry variations are generated by $\delta_{\epsilon}=\bar{\epsilon} \mathcal{Q}-\epsilon \overline{\mathcal{Q}}$. We may easily read off the following transformation rules of the component fields

$$
\delta \phi=i \bar{\epsilon} \psi-i \bar{\psi} \epsilon, \quad \delta \psi=(\dot{\phi}-i F) \epsilon, \quad \delta \bar{\psi}=\bar{\epsilon}(\dot{\phi}+i F), \quad \delta F=-\bar{\epsilon} \dot{\psi}-\dot{\bar{\psi}} \epsilon
$$

by acting with the supersymmetry variations on the superfield:

$$
\delta_{\epsilon} \Phi=\bar{\epsilon}(i \psi+i \theta F+\theta \dot{\phi}-\bar{\theta} \theta \dot{\psi})-(i \bar{\psi}+i \bar{\theta} F-\bar{\theta} \dot{\phi}+\bar{\theta} \theta \dot{\bar{\psi}}) \epsilon .
$$

Here and in the following, a dot denotes differentiation w.r.t. $\tau$. In order to obtain a supersymmetric action, we further need the supercovariant derivatives $D=i \partial_{\bar{\theta}}-\theta \partial_{\tau}$ and $\bar{D}=i \partial_{\theta}-\bar{\theta} \partial_{\tau}$. They fulfill almost identical anticommutation relations as the supercharges,

$$
\{D, D\}=\{\bar{D}, \bar{D}\}=0 \quad \text { and } \quad\{D, \bar{D}\}=-2 H,
$$

and anticommute with the supercharges. With these definitions, one can write down the supersymmetric Euclidean off-shell action within the superfield formalism:

$$
S[\phi, F, \bar{\psi}, \psi]=\int \mathrm{d} \tau \mathrm{d} \theta \mathrm{d} \bar{\theta}\left[-\frac{1}{2} \Phi K \Phi+i W(\Phi)\right]
$$




$$
=\int \mathrm{d} \tau\left[\frac{1}{2} \dot{\phi}^{2}-i \bar{\psi} \dot{\psi}+i F W^{\prime}(\phi)-i \bar{\psi} W^{\prime \prime}(\phi) \psi+\frac{1}{2} F^{2}\right],
$$

where we introduced the kinetic operator

$$
K=\frac{1}{2}(\bar{D} D-D \bar{D})
$$

A prime always denotes the derivative with respect to the scalar field $\phi$. Eliminating the auxiliary field $F$ by its equation of motion, $F=-i W^{\prime}$, we obtain the on-shell action

$$
S_{\text {on }}[\phi, \psi, \bar{\psi}]=\int \mathrm{d} \tau\left[\frac{1}{2} \dot{\phi}^{2}-i \bar{\psi} \dot{\psi}+\frac{1}{2} W^{\prime 2}(\phi)-i W^{\prime \prime}(\phi) \bar{\psi} \psi\right] .
$$

From (2.9) we read off the bosonic potential $V(\phi)=\frac{1}{2} W^{\prime 2}(\phi)$ and the Yukawa term $W^{\prime \prime} \bar{\psi} \psi$. If supersymmetry is unbroken, the ground state energy vanishes.

Let us assume the superpotential $W(\phi)$ to be a polynomial in the scalar field. Then, the global properties of the superpotential $W(\phi) \sim \phi^{n}$ for large $\phi$ determine whether spontaneous breaking of supersymmetry occurs or not. If $n$ is even, supersymmetry will be intact on all scales. This is realized e.g. for quartic classical superpotentials

$$
W(\phi)=e \phi+\frac{m}{2} \phi^{2}+\frac{g}{3} \phi^{3}+\frac{a}{4} \phi^{4},
$$

which we will consider in section 2.2.2. $W(\phi)$ represents the microscopic superpotential, i.e. the initial potential of our quantum system before fluctuations are taken into account. If $n$ is odd, the effective potential exhibits a ground state with positive energy and supersymmetry is spontaneously broken, even if we may start with a microscopic potential with vanishing ground state energy. This applies e.g. to cubic classical superpotentials of the form

$$
W(\phi)=e \phi+\frac{g}{3} \phi^{3}, \quad e<0, g>0,
$$

which will be discussed in detail in section 2.3.

\subsection{Flow equation in superspace}

In order to analyze supersymmetric quantum mechanical systems, we resort to Wilsonian renormalization group techniques. Specifically, we adopt the framework of the FRG, formulated in terms of a flow equation for the effective average action $\Gamma_{k}$. It is based on the infinitesimal integrating-out of degrees of freedom with momenta larger than some infrared momentum scale $k^{2}$. Thus, at a certain scale $k$ all quantum fluctuations with momenta $|p|>k$ are taken into account. Hence, $\Gamma_{k}$ interpolates between the microscopic action $S$ in the ultraviolet and the full quantum effective action $\Gamma_{k \rightarrow 0}=\Gamma$ in the infrared (IR). It obeys the exact functional differential equation [9]

$$
\partial_{k} \Gamma_{k}=\frac{1}{2} \mathrm{~S} \operatorname{Tr}\left\{\left[\Gamma_{k}^{(2)}+R_{k}\right]^{-1} \partial_{k} R_{k}\right\} .
$$

This flow equation reads in superspace

$$
\partial_{k} \Gamma_{k}=\frac{1}{2} \int \mathrm{d} z \mathrm{~d} z^{\prime} \partial_{k} R_{k}\left(z, z^{\prime}\right) G_{k}\left(z^{\prime}, z\right), \quad G_{k}=\left(\Gamma_{k}^{(2)}+R_{k}\right)^{-1},
$$


where $z=(\tau, \theta, \bar{\theta})$ denotes the coordinates in superspace. Therein the second functional derivative with respect to the superfield $\Gamma_{k}^{(2)}$ is given by

$$
\left(\Gamma_{k}^{(2)}\right)\left(z, z^{\prime}\right)=\frac{\vec{\delta}}{\delta \Phi(z)} \Gamma_{k} \frac{\overleftarrow{\delta}}{\delta \Phi\left(z^{\prime}\right)}
$$

Note that the supertrace in (2.12) as well as the right- and left-derivatives in (2.14) take care of the minus signs for anticommuting variables.

\subsubsection{Supercovariant derivative expansion in NNLO}

In this work, we employ the expansion of $\Gamma_{k}$ in powers of the supercovariant derivatives $D$ and $\bar{D}$ with mass-dimension $1 / 2$. Unfortunately, a systematic and consistent expansion scheme of $\Gamma_{k}$ does not guarantee convergence. One goal of the present work is to demonstrate the convergence of the supercovariant derivative expansion at NNLO to numerically known values of observables. We will derive the flow equation in the off-shell formulation with a manifestly supersymmetric regulator such that in each order of the supercovariant derivative expansion the flow preserves supersymmetry.

To this order, the most general ansatz for the scale-dependent effective action reads

$$
\Gamma_{k}[\Phi]=\int \mathrm{d} z\left[i W_{k}(\Phi)-\frac{1}{2} Z_{k}(\Phi) K Z_{k}(\Phi)+\frac{i}{4} Y_{1, k}(\Phi) K^{2} \Phi+\frac{i}{4} Y_{2, k}(\Phi)(K \Phi)(K \Phi)\right],
$$

with the scale and field dependent functions $W_{k}, Z_{k}, Y_{1, k}$ and $Y_{2, k}$ and the kinetic operator $K$ introduced in (2.8). A contribution to $\Gamma_{k}$ where the derivatives act on three superfields, $Y_{3, k}(\bar{D} \Phi)(D \Phi)(K \Phi)$, is already included in our truncation, since

$$
\int \mathrm{d} z A(\Phi) K^{2} \Phi=\int \mathrm{d} z\left[A^{\prime}(\Phi)(K \Phi)(K \Phi)+A^{\prime \prime}(\Phi)(\bar{D} \Phi)(D \Phi)(K \Phi)\right] .
$$

Terms with $D, \bar{D}$ acting on four superfields do not exist since $(\bar{D} \Phi D \Phi)^{2}=0$. In component fields the action (2.15) takes the form ${ }^{1}$

$$
\begin{aligned}
\Gamma_{k}[\Phi]= & \int \mathrm{d} \tau\left[\frac{1}{2} Z^{\prime 2} \dot{\phi}^{2}-i Z^{\prime 2} \bar{\psi} \dot{\psi}-\frac{i}{2}\left(Y_{1}^{\prime}+Y_{2}\right) \dot{\bar{\psi}} \dot{\psi}-i\left(W^{\prime \prime}+Z^{\prime} Z^{\prime \prime} \dot{\phi}-\frac{1}{2} Y_{1}^{\prime \prime} \ddot{\phi}-\frac{1}{4} Y_{1}^{\prime \prime \prime} \dot{\phi}^{2}\right) \bar{\psi} \psi\right. \\
& +\left(i W^{\prime}-Z^{\prime} Z^{\prime \prime} \bar{\psi} \psi-\frac{i}{2}\left(Y_{1}^{\prime}+Y_{2}\right) \ddot{\phi}-\frac{i}{4} Y_{1}^{\prime \prime} \dot{\phi}^{2}+\frac{1}{2} Y_{2}^{\prime}(\bar{\psi} \dot{\psi}-\dot{\bar{\psi}} \psi)\right) F \\
& \left.+\left(\frac{1}{2} Z^{\prime 2}-\frac{i}{4} Y_{2}^{\prime \prime} \bar{\psi} \psi\right) F^{2}+\frac{i}{4} Y_{2}^{\prime} F^{3}\right]
\end{aligned}
$$

where the terms are ordered according to increasing powers of the auxiliary field $F$.

\subsubsection{Introducing the regulator functional}

The flow of $\Gamma_{k}$ is regularized by adding a suitable regulator functional $\Delta S_{k}$ to the action, in such a way that $R_{k}=\Delta S_{k}^{(2)}$. Given a supersymmetric truncation $\Gamma_{k}$ and a supersymmetric

\footnotetext{
${ }^{1}$ For convenience, we often omit the index $k$ and the explicit dependence on the superfield $\Phi$ of the scalar functions $W_{k}, Z_{k}, Y_{1, k}$ and $Y_{2, k}$ from now on.
} 
initial condition, we only need a supersymmetric regulator in order to construct a manifestly supersymmetric flow. Following [15-17], the most general off-shell supersymmetric cutoff action quadratic in the superfields can be written as

$$
\Delta S_{k}=\frac{1}{2} \int \mathrm{d} z \Phi R_{k}(D, \bar{D}) \Phi
$$

As $D$ and $\bar{D}$ satisfy the anticommutation relation (2.6), it can be written as

$$
\Delta S_{k}=\frac{1}{2} \int \mathrm{d} z \Phi\left[i r_{1}\left(-\partial_{\tau}^{2}, k\right)-Z^{\prime 2}(\bar{\Phi}) r_{2}\left(-\partial_{\tau}^{2}, k\right) K\right] \Phi,
$$

where $Z^{\prime}$ is evaluated at the background field $\bar{\Phi}=\bar{\phi}$. Thus, in momentum space $R_{k}$ is given by $^{2}$

$$
R_{k}\left(q, q^{\prime}, \theta, \theta^{\prime}\right)=\left[i r_{1}\left(q^{2}, k\right)-Z^{\prime 2}(\bar{\phi}) r_{2}\left(q^{2}, k\right) K(q, \theta)\right] \delta\left(q, q^{\prime}\right) \delta\left(\theta, \theta^{\prime}\right) .
$$

The regulator function $r_{1}$ with mass dimension 1 acts like an additional momentumdependent mass and ensures a gap $\sim k$ for the IR modes. Note that we do not spectrally adjust this regulator function by multiplying it with the wave function renormalization as has been done in [15]. The latter approach would actually slow down the flow of the higher order operators $Z, Y_{1}, Y_{2}$. The dimensionless regulator function $r_{2}$ can be viewed as a deformation of the momentum dependence of the kinetic term. The term $q^{2} r_{2}\left(q^{2} / k^{2}\right)$ represents the supersymmetric analogue of the corresponding regulator function $r_{k}\left(q^{2} / k^{2}\right)$ in scalar field theory [10]. Here, a spectral adjustment via the inclusion of the wave function renormalization $Z^{\prime}(\bar{\Phi})$ is helpful in order to provide a simple form for the flow of $\Gamma_{k}$ [30]. We did check the influence of the spectral adjustment on the flow of $\Gamma_{k}$ carefully.

\subsubsection{Flow equation}

We begin with the calculation of the second functional derivative of $\Gamma_{k}$ as defined in (2.14) in order to derive its flow according to (2.13). We find ${ }^{3}$

$$
\begin{gathered}
\left(\Gamma_{k}^{(2)}+R_{k}\right)\left(z, z^{\prime}\right)=\left[i\left(W^{\prime \prime}+r_{1}\right)-Z^{\prime \prime}(K Z)-Z^{\prime} K Z^{\prime}-Z^{\prime}(\bar{\Phi})^{2} r_{2} K+\frac{i}{4}\left\{Y_{1}^{\prime \prime}\left(K^{2} \Phi\right)\right.\right. \\
\left.\left.+Y_{1}^{\prime} K^{2}+K^{2} Y_{1}^{\prime}+Y_{2}^{\prime \prime}(K \Phi)^{2}+2 Y_{2}^{\prime}(K \Phi) K+2 K Y_{2}^{\prime}(K \Phi)+2 K Y_{2} K\right\}\right] \delta\left(z, z^{\prime}\right) .
\end{gathered}
$$

The scale dependent functions $W, Z, Y_{1}, Y_{2}$ are functions of the superfield $\Phi(z)$ whereas the scale dependent $Z^{\prime}(\bar{\Phi})$ has the background field as argument. Here, a bracket implies that the kinetic operator $K$ only acts within the bracket. If there is no bracket, then it acts on everything on its right hand side.

We may derive the flow of the scalar functions $W^{\prime}(\phi), Z^{\prime}(\phi)$ and $Y_{2}^{\prime}(\phi)$ via a projection onto the coefficients of $F, F^{2}$ and $F^{3}$ in (2.17) with fermionic fields and time derivatives set

\footnotetext{
${ }^{2}$ We abbreviate $\delta\left(\theta, \theta^{\prime}\right):=\delta\left(\bar{\theta}-\bar{\theta}^{\prime}\right) \delta\left(\theta-\theta^{\prime}\right)$.

${ }^{3}$ The functional derivative w.r.t. the superfields is defined such that $\int d z \frac{\delta \Phi(z)}{\delta \Phi\left(z^{\prime}\right)}=\int d z \delta\left(z, z^{\prime}\right)=1$, where $\delta\left(z, z^{\prime}\right):=\delta\left(\tau, \tau^{\prime}\right) \delta\left(\theta, \theta^{\prime}\right)$.
} 
to zero. Thus, it suffices to consider constant component fields in (2.21) and set $\bar{\psi}=\psi=0$ afterwards. Switching to momentum space, the inverse propagator (2.21) takes the form

$$
\begin{aligned}
& \left(\Gamma_{k}^{(2)}+R_{k}\right)\left(q, q^{\prime}, \theta, \theta^{\prime}\right)=\left[\left(i\left(W^{\prime \prime}+r_{1}\right)+Z^{\prime} Z^{\prime \prime} F+\frac{i}{2}\left(Y_{1}^{\prime}+Y_{2}\right) q^{2}+\frac{i}{4} Y_{2}^{\prime \prime} F^{2}\right) \delta\left(\theta, \theta^{\prime}\right)\right. \\
& +\left(i W^{\prime \prime \prime} F+\left(Z^{\prime} Z^{\prime \prime \prime}+Z^{\prime \prime 2}\right) F^{2}+B q^{2}+\frac{i}{4} Y_{2}^{\prime \prime \prime} F^{3}+i\left(\frac{1}{2} Y_{1}^{\prime \prime}+Y_{2}^{\prime}\right) F q^{2}\right) \bar{\theta} \theta \bar{\theta}^{\prime} \theta^{\prime} \\
& \left.+\left(B+\frac{3}{2} i F Y_{2}^{\prime}\right)+\left(B q+i F Y_{2}^{\prime} q\right)\left(\bar{\theta}^{\prime} \theta-\bar{\theta} \theta^{\prime}\right)+\left(Z^{\prime} Z^{\prime \prime} F+\frac{i}{2} Y_{2}^{\prime \prime} F^{2}\right)\left(\bar{\theta} \theta+\bar{\theta}^{\prime} \theta^{\prime}\right)\right] \delta\left(q, q^{\prime}\right),
\end{aligned}
$$

where the background field enters via $B=Z^{\prime 2}+r_{2} Z^{\prime 2}(\bar{\phi})$. The Greens function in superspace, $G_{k}=\left(\Gamma_{k}^{(2)}+R_{k}\right)^{-1}$, is determined by

$$
\int \frac{\mathrm{d} q^{\prime}}{2 \pi} \mathrm{d} \theta^{\prime} \mathrm{d} \bar{\theta}^{\prime} G_{k}^{-1}\left(q, q^{\prime}, \theta, \theta^{\prime}\right) G_{k}\left(q^{\prime}, q^{\prime \prime}, \theta^{\prime}, \theta^{\prime \prime}\right)=\delta\left(q, q^{\prime \prime}\right) \delta\left(\theta, \theta^{\prime \prime}\right) .
$$

To continue, we make the general ansatz

$$
G_{k}\left(q, q^{\prime}, \theta, \theta^{\prime}\right)=\left(a+b \bar{\theta} \theta+c \bar{\theta}^{\prime} \theta^{\prime}+d \bar{\theta} \theta^{\prime}+e \bar{\theta}^{\prime} \theta+f \bar{\theta} \theta \bar{\theta}^{\prime} \theta^{\prime}\right) \delta\left(q, q^{\prime}\right),
$$

with arbitrary coefficients depending on the scalar functions in (2.22) and the momentum $q$. By solving (2.23) for the coefficients in $G_{k}$, the projected flow equation reads:

$$
\begin{aligned}
& \left.\partial_{k} \Gamma_{k}\right|_{\dot{\phi}=\dot{F}=\psi=\bar{\psi}=0}=\int \mathrm{d} \tau\left(i \partial_{k} W^{\prime} F+\frac{1}{2} \partial_{k} Z^{\prime 2} F^{2}+\frac{i}{4} \partial_{k} Y_{2}^{\prime} F^{3}\right) \\
& =\frac{1}{2} \int \frac{\mathrm{d} q}{2 \pi} \frac{\mathrm{d} q^{\prime}}{2 \pi} \mathrm{d} \theta \mathrm{d} \bar{\theta} \mathrm{d} \theta^{\prime} \mathrm{d} \bar{\theta}^{\prime}\left(\partial_{k} R_{k}\right)\left(q^{\prime}, q, \theta^{\prime}, \theta\right) G_{k}\left(q, q^{\prime}, \theta, \theta^{\prime}\right) \\
& =\frac{1}{2} \int \mathrm{d} \tau \frac{\mathrm{d} q}{2 \pi}\left[i\left(\partial_{k} r_{1}\right)(b+c+d+e)+\partial_{k}\left(r_{2} Z^{\prime 2}(\bar{\phi})\right)\left(f+a q^{2}-e q+d q\right)\right] .
\end{aligned}
$$

The solutions for the coefficients are presented in appendix A. By extracting the coefficients of $F, F^{2}$ and $F^{3}$ on the right hand side we obtain the flow equations for $W^{\prime}, Z^{\prime}$ and $Y_{2}^{\prime}$. Since the flow of $Y_{1}$ is missing, we further project the flow equation onto the coefficient of $\dot{F} \dot{\phi}$. This way, one obtains the flow of $Y_{1}^{\prime}(\phi)+Y_{2}(\phi)$ and thus a closed system of four coupled PDE's. Appendix B contains the detailed derivation of this flow which requires the inverse propagator for momentum-dependent fields $\phi, F$. To simplify the obtained flow equations, we define the new functions

$$
Y:=Y_{2}^{\prime} \quad \text { and } \quad X:=Y_{1}^{\prime}+Y_{2} .
$$

It remains to solve the flow equations for the scale dependent functions $W^{\prime}, Z^{\prime}, X$ and $Y$.

\subsection{Effective potential and first excited energy}

The low lying energies can be extracted from the bosonic on-shell effective potential $V_{\text {eff }}=$ $V_{k=0}$. In order to compute $V_{k}$, we set the fermionic fields to zero in the truncated effective average action (2.17). 


\subsubsection{On-shell effective potential}

At a given scale, the auxiliary field $F$ fulfills the equation of motion

$$
F=-\frac{2 i}{3 Y}\left(\sqrt{Z^{\prime 4}+\frac{3}{4}\left(4 W^{\prime}-2 X \ddot{\phi}-\left(X^{\prime}-Y\right) \dot{\phi}^{2}\right) Y}-Z^{\prime 2}\right),
$$

and hence becomes dynamical, in contrast to the situation in the NLO approximation. Next, we eliminate the auxiliary field in the bosonic action by its equation of motion. To calculate the effective potential $V_{\text {eff }}$, it is sufficient to consider $\Gamma_{k}[\phi]$ for constant $\phi$ in which case

$$
V_{k}(\phi)=\frac{2}{27 Y^{2}}\left(\sqrt{3 W^{\prime} Y+Z^{\prime 4}}-Z^{\prime 2}\right)\left(6 W^{\prime} Y+Z^{\prime 4}-Z^{\prime 2} \sqrt{3 W^{\prime} Y+Z^{\prime 4}}\right) .
$$

We determine the energy of the first excited state $E_{1}$ from the propagator $G_{k}$ at vanishing $k$, where the regulator $R_{k}$ vanishes. Supersymmetry is unbroken if the potential $V_{k}$ in (2.28) vanishes at its minimum $\phi_{\min }$, which is the case if $W_{k}^{\prime}\left(\phi_{\min }\right)=0$. Actually, in the strong coupling regime there exists a second solution for which $\left[4 W^{\prime}\left(Y+Z^{\prime 4}\right)\right]\left(\phi_{\min }\right)=0$. However, we believe this solution to be unphysical, see section 2.2.2.

For a constant $\phi_{\min }$, the auxiliary field $F$ in $(2.27)$ vanishes if $W_{k}^{\prime}\left(\phi_{\min }\right)=0$. Thus, we determine the excited energies $E_{1}$ by considering the propagator (2.24) for constant fields $\phi$ and $W^{\prime}=F=0$. After an integration over the Grassmann variables, we obtain

$$
\left.G_{k}\left(q, q^{\prime}, \theta, \theta^{\prime}\right)\right|_{\bar{\theta} \theta \bar{\theta}^{\prime} \theta^{\prime}}=\frac{Z^{\prime 2} q^{2}}{Z^{\prime 4} q^{2}+\left(W^{\prime \prime}+1 / 2 X q^{2}\right)^{2}} \delta\left(q-q^{\prime}\right) .
$$

The square of the excited energy $E_{1}^{2}$ is then given by the pole of the propagator at the minimum of the effective potential:

$$
\left.\lim _{k \rightarrow 0}\left(Z^{\prime 4} q_{0}^{2}+\left(W^{\prime \prime}+\frac{1}{2} X q_{0}^{2}\right)^{2}\right)\right|_{\phi_{\min }}=0 \quad \text { with } \quad q_{0}^{2}=\left(i E_{1}\right)^{2} .
$$

This equation possesses the two solutions

$$
E_{1}^{2}=\left.\lim _{k \rightarrow 0} \frac{2}{X^{2}}\left(Z^{\prime 4}+X W^{\prime \prime} \pm Z^{\prime 2} \sqrt{Z^{\prime 4}+2 X W^{\prime \prime}}\right)\right|_{\phi_{\min }},
$$

where the solution with the negative sign is the correct one, since it reduces to the known limiting value $E_{1}=\left|W^{\prime \prime}\left(\phi_{\min }\right)\right|$ in the LPA approximation with $Z^{\prime}=1$ and $X=0$. The other solution with positive sign diverges in this limit.

Note that if supersymmetry is spontaneously broken, $W_{k}^{\prime}\left(\phi_{\min }\right) \neq 0$ and the corresponding auxiliary field $F$ does not vanish. Then the first excited energy $E_{1}$ is extracted from the pole of the general propagator (2.24), i.e. of

$$
\left.\lim _{k \rightarrow 0} G_{k}\left(q, q^{\prime}, \theta, \theta^{\prime}\right)\right|_{\bar{\theta} \theta \bar{\theta}^{\prime} \theta^{\prime}}
$$

at the (constant) minimum $\phi_{\min }$ of the potential, where $F$ has to be replaced by its equation of motion (2.27). 


\subsubsection{Numerical results}

The flow equation of the superpotential in NNLO in the derivative expansion is given in its full form by

$$
\begin{aligned}
& \partial_{k} W_{k}^{\prime}(\phi)=\frac{1}{2} \int_{-\infty}^{\infty} \frac{\mathrm{d} q}{2 \pi} {\left[\partial_{k} r_{1} \frac{2\left(Z^{\prime} Z^{\prime \prime}\left(A^{2}-B^{2} q^{2}\right)-B A A^{\prime}\right)}{\left(B^{2} q^{2}+A^{2}\right)^{2}}\right.} \\
&\left.+\partial_{k}\left(r_{2} Z^{\prime 2}(\bar{\phi})\right) \frac{\left(A^{\prime}\left(A^{2}-B^{2} q^{2}\right)+4 B q^{2} A Z^{\prime} Z^{\prime \prime}\right)}{\left(B^{2} q^{2}+A^{2}\right)^{2}}\right]
\end{aligned}
$$

where $A=W^{\prime \prime}+r_{1}+\frac{q^{2}}{2} X$ and as earlier $B=Z^{\prime 2}+r_{2} Z^{\prime 2}(\bar{\phi})$. Now, we specify the regulator functions and choose $r_{2}\left(q^{2}, k\right)=0$ and the Callan-Symanzik regulator $r_{1}\left(q^{2}, k\right)=k$. Then there is no dependence on the background field and the flow equation of $W^{\prime}(\phi)$ simplifies to

$$
\partial_{k} W^{\prime}(\phi)=\frac{Z^{\prime}}{4 \mathcal{W}^{\prime \prime 2}} \frac{\mathcal{W}^{\prime 2}\left(X^{\prime} Z^{\prime}+4 X Z^{\prime \prime}\right)-\mathcal{W}^{\prime \prime \prime} Z^{\prime}\left(3 \mathcal{W}^{\prime \prime} X+Z^{\prime 4}\right)}{\left(2 \mathcal{W}^{\prime \prime} X+Z^{\prime 4}\right)^{3 / 2}}, \quad \mathcal{W}^{\prime \prime}=W^{\prime \prime}+k
$$

where the momentum integration has been performed. Note that the right hand side of the flow equations only depends via $W^{\prime \prime}$ and $W^{\prime \prime \prime}$ on the superpotential. The corresponding microscopic action in the UV is given by (2.9) and we focus on quartic superpotentials of the form (2.10). Thus, the initial conditions for the flow at $k=\Lambda$ read

$$
W_{\Lambda}^{\prime}(\phi)=e+m \phi+g \phi^{2}+a \phi^{3}, \quad Z_{\Lambda}^{\prime}(\phi)=1, \quad Y_{\Lambda}(\phi)=X_{\Lambda}(\phi)=0 .
$$

In supersymmetric quantum mechanics the fluctuations in the UV are suppressed and the flow freezes out for $k \rightarrow \Lambda \rightarrow \infty$. Hence the initial conditions are stable for large UV-cutoffs. Indeed, plugging (2.35) into the flow equations yields

$$
\left.\partial_{k} W^{\prime}\right|_{\Lambda}=O\left(\Lambda^{-2}\right),\left.\quad \partial_{k} Z^{\prime}\right|_{\Lambda}=O\left(\Lambda^{-4}\right),\left.\quad \partial_{k} X\right|_{\Lambda}=O\left(\Lambda^{-5}\right),\left.\quad \partial_{k} Y\right|_{\Lambda}=O\left(\Lambda^{-6}\right) .
$$

For $W_{\Lambda}^{\prime}$ in (2.35) supersymmetry remains unbroken at all scales. Note that the initial superpotential $W_{\Lambda}$ is non-convex if $g^{2}>3 m a$. Besides, we may shift the field $\phi \rightarrow \phi-g /(3 a)$ such that the quadratic term of $W_{\Lambda}^{\prime}$ vanishes.

We solved the set of the four coupled partial differential equations for $W^{\prime}, Z^{\prime}, X, Y$ numerically with spectral methods (see appendix D). Besides, we repeated the calculations with the implicit Runge-Kutta method of NDSolve of MATHEMATICA 9. Here, we have chosen $\phi \in(-100,100)$ and kept the four functions at their classical values at the boundary for all scales as the flows vanish for $|\phi| \rightarrow \infty$. With both methods we obtained the same results to three or four significant digits.

Table 1 displays the energy gap $E_{1}(g)$ for $e=a=m=1$ and various values of the coupling $g$. We also listed the resulting energies obtained by solving the PDE's in LPA (includes $\left.W_{k}(\phi)\right)$ as well as NLO (includes $W_{k}(\phi)$ and $Z_{k}(\phi)$ ).

Figure 1 shows the first excited energies $E_{1}$ (left figure) and the relative deviation from the exact values $e_{\text {trunc }}=\left(E_{1}-E_{1}^{\text {ex }}\right) / E_{1}^{\text {ex }}$ (right figure) as a function of the coupling $g$. Obviously, an inclusion of terms of fourth-order in the derivative expansion improves the 

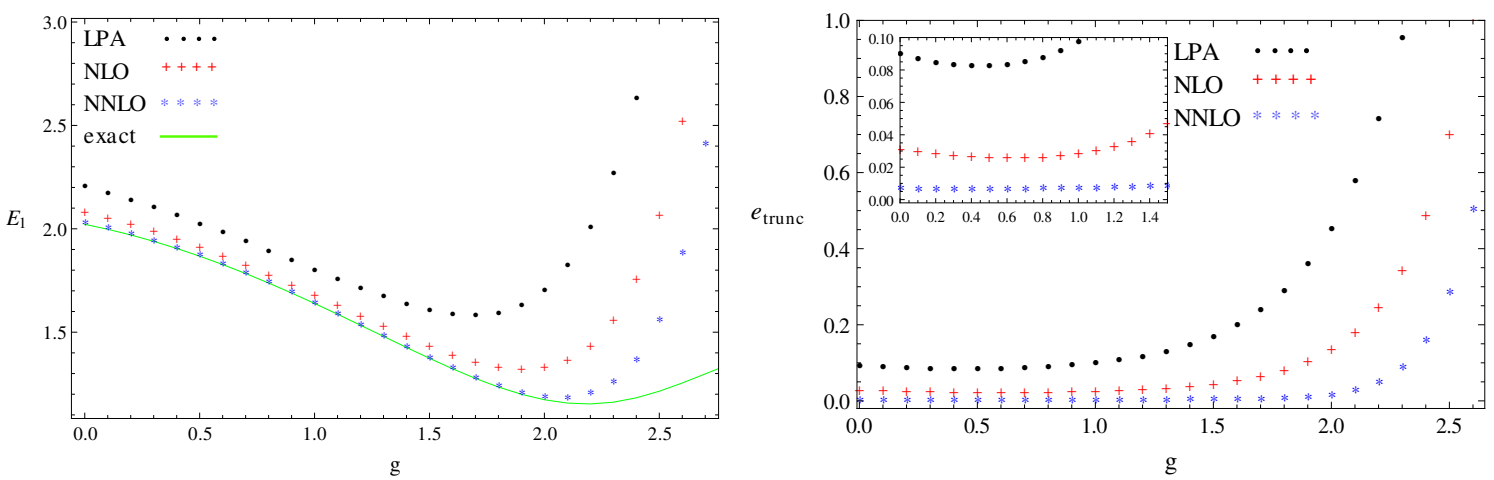

Figure 1. Energy gap $E_{1}(g)$ and relative error $e_{\text {trunc }}$ for classical superpotentials of the form $W_{\Lambda}^{\prime}(\phi)=1+\phi+g \phi^{2}+\phi^{3}$ and various $g$. For convex initial potentials $(g<\sqrt{3})$ we achieve a nice convergence as well as a relative error of $1 \%$ in NNLO. For couplings larger than $g \approx 2$, where the classical potential becomes non-convex, we observe significant deviations from the exact results.

\begin{tabular}{|llllc|}
\hline$g$ & $E_{1}^{\mathrm{LPA}}$ & $E_{1}^{\mathrm{NLO}}$ & $E_{1}^{\mathrm{NNLO}}$ & $E_{1}^{\mathrm{ex}}$ \\
\hline 0.0 & 2.202 & 2.086 & 2.038 & 2.022 \\
0.2 & 2.136 & 2.028 & 1.986 & 1.970 \\
0.4 & 2.061 & 1.957 & 1.920 & 1.905 \\
0.6 & 1.978 & 1.876 & 1.842 & 1.827 \\
0.8 & 1.889 & 1.784 & 1.752 & 1.738 \\
1.0 & 1.797 & 1.687 & 1.653 & 1.639 \\
1.2 & 1.709 & 1.584 & 1.547 & 1.534 \\
1.4 & 1.632 & 1.486 & 1.440 & 1.426 \\
1.6 & 1.583 & 1.398 & 1.337 & 1.323 \\
1.8 & 1.590 & 1.339 & 1.250 & 1.235 \\
2.0 & 1.702 & 1.337 & 1.199 & 1.173 \\
2.2 & 2.005 & 1.442 & 1.216 & 1.153 \\
2.4 & 2.627 & 1.764 & 1.378 & 1.183 \\
2.6 & 3.661 & 2.525 & 1.895 & 1.254 \\
2.8 & 4.988 & 3.961 & 3.195 & 1.343 \\
\hline
\end{tabular}

Table 1. Energy $E_{1}^{\mathrm{NNLO}}$ of the first excited state, calculated according to (2.31) for $r_{1}=k$, $e=m=a=1$ and various $g$. For comparison, also the results $E_{1}^{\mathrm{LPA}}$ obtained in LPA, $E_{1}^{\mathrm{NLO}}$ derived in NLO as well as the exact values $E_{1}^{\text {ex }}$ from numerically diagonalizing the Hamiltonian are given. Here, $E_{1}^{\mathrm{LPA}}, E_{1}^{\mathrm{NLO}}$ and $E_{1}^{\mathrm{NNLO}}$ were derived by solving the respective partial differential equations numerically. 

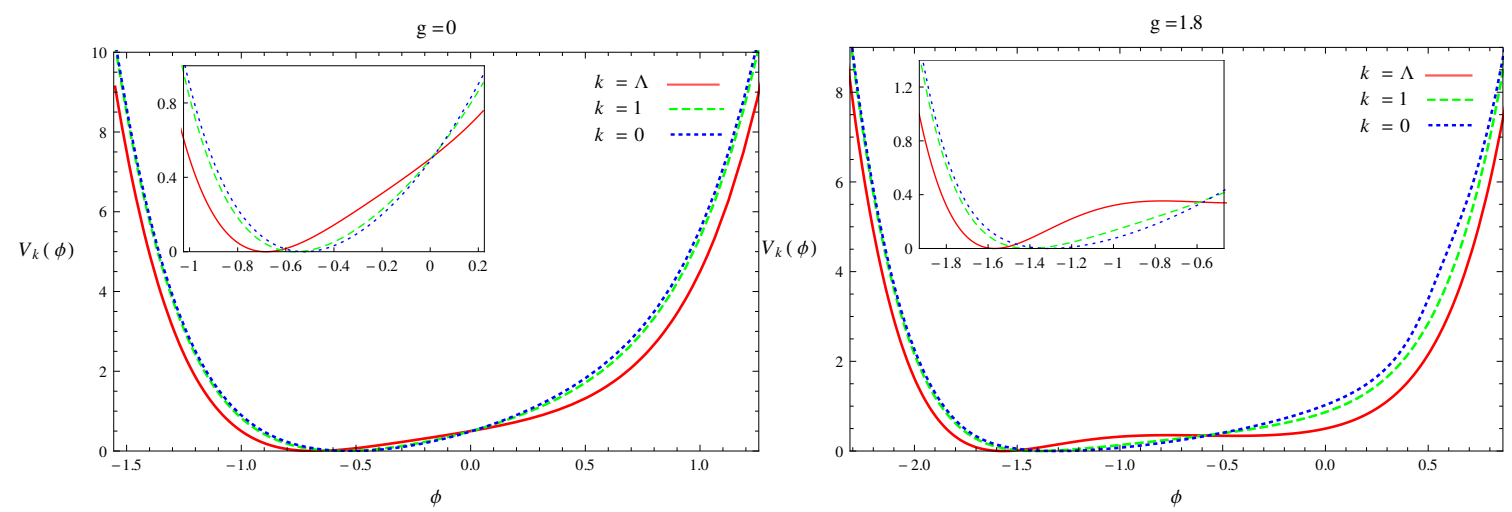

Figure 2. Flow of the effective average potential $V_{k}(\phi)$ as obtained by solving the system of PDE's numerically in NNLO in the derivative expansion with initial conditions (2.35), where $e=m=a=1$ and $g=0$ (left panel) and $g=1.8$ (right panel).

results for the energy gap considerably. We obtain a relative error of $<1 \%$ for couplings $g<\sqrt{3}$. For couplings $\sqrt{3}<g<2.3$ the relative deviations from the exact results lie within a $10 \%$ error.

Note that for couplings larger than $g \approx 2$ the error increases exponentially and the supercovariant derivative approximation breaks down. The breakdown of the NNLO approximation for couplings $g \gtrsim 2$ is also indicated by the structure of the effective average potential. In this regime, $V_{k}(\phi)$ becomes complex for all scales smaller than a $k_{0}>0$ for field values close to the local minimum of $W_{\Lambda}^{\prime}$. This is due to the expression $\sqrt{3 W^{\prime} Y+Z^{\prime 4}}$ appearing in (2.28) which becomes complex near the local minimum of $W_{\Lambda}^{\prime}$ for non-convex initial potentials, owing to an increasingly negative $Y$; see also figures 3,4 . Another sign of the breakdown is given by the appearance of a further mass at $g \approx 1.7$, splitting in two masses for even larger couplings $g$. This is due to the formation of one/two further minima of the effective potential, where $4 W^{\prime} Y+\left.Z^{\prime 4}\right|_{\phi_{\min }}=0$ holds. Here, the fourth order correction $Y$ is of the same order as the leading and next-to leading order terms $W^{\prime}$ and $Z^{\prime}$ indicating the invalidity of the truncation. The corresponding masses become parametrically large. These large masses in the strong coupling regime are probably an artifact of the regularization and have no physical significance. Similar artifacts have already been encountered in $O(N)$ symmetric Wess-Zumino models [24].

We thus observe a very good convergence of the derivative expansion in case the local barrier of the classical potential is small. However, as the non-convexity of $V_{\Lambda}$ increases, tunneling events are exponentially suppressed and are no longer captured by the flow equations in the derivative expansion. Here, the inclusion of non-local operators should lead to a better convergence behaviour in the strong coupling regime.

The flow of the bosonic potential $V_{k}(\phi)$ for $g=0$ and $g=1.8$ is depicted in figure 2 . Apparently, non-convexities appearing in the classical potential diminish as more and more long-range quantum fluctuations are taken into account such that the effective potentials in figure 2 become convex. ${ }^{4}$ Furthermore, figures 3 and 4 show the flow of $W^{\prime}, Z^{\prime}, X$ and

\footnotetext{
${ }^{4}$ However, note that the structure of the flow equation (2.12) forces rather the superpotential than the scalar potential to become convex in the IR. Since the scalar potential is a complicated function of $W^{\prime}, Z, Y$, i.e. of the form (2.28), the flow equation does not immediately imply $V_{k \rightarrow 0}$ to be convex.
} 


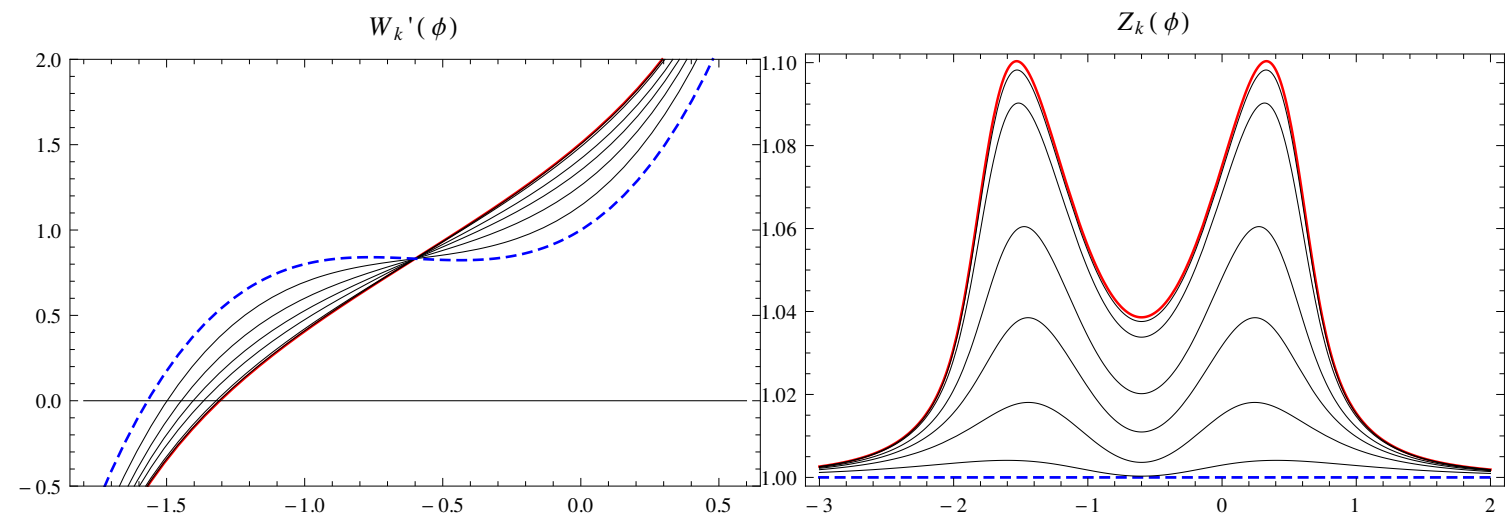

Figure 3. The superpotential $W_{k}^{\prime}(\phi)$ and the wave function renormalization $Z_{k}^{\prime}(\phi)$ for different scales $k$. Starting in the $U V$ at $k=\Lambda=10^{4}$ (blue, dashed line) the flow evolves to the IR at $k=0$ (red solid line). The intermediate scales are $k=5,2,1,0.5,0.1,0.02$.
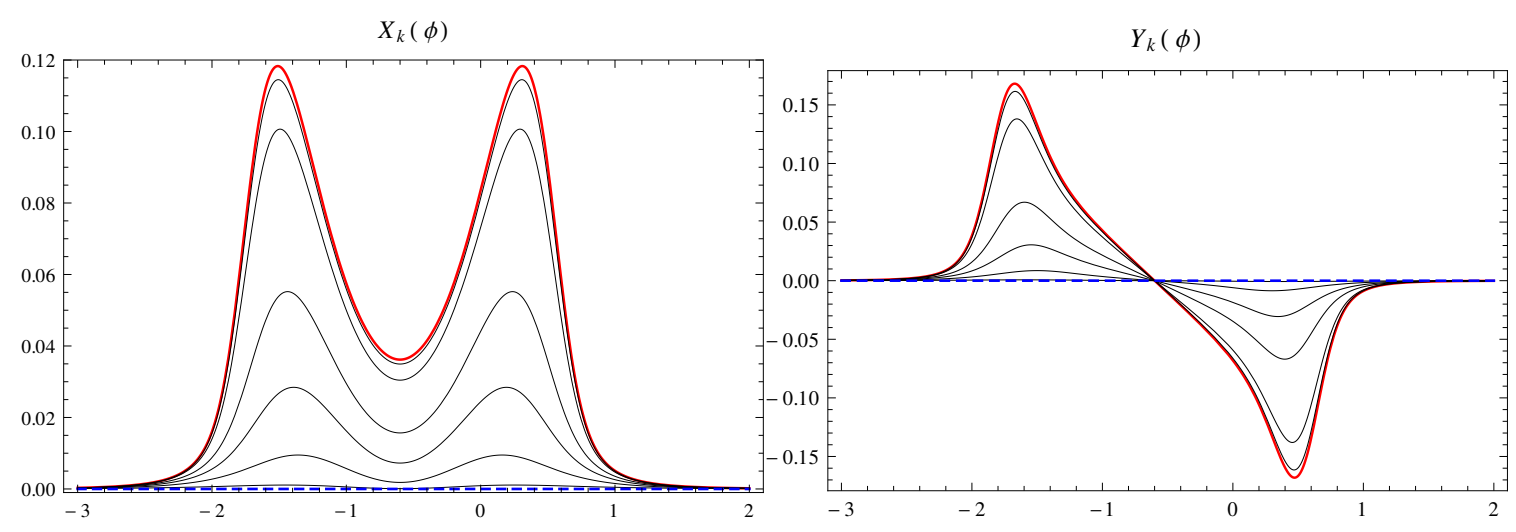

Figure 4. The flow of the fourth-order couplings $X_{k}(\phi), Y_{k}(\phi)$ for different scales $k$. Starting in the $U V$ at $k=\Lambda=10^{4}$ (blue, dashed line) the flow evolves to the IR at $k=0$ (red solid line). The intermediate scales are $k=5,2,1,0.5,0.1,0.02$.

$Y$ for $g=1.8$. From (2.36) we infer the following deviation of the solutions at $k=0$ from their classical values for large values of $|\phi|$ :

$$
W_{0}^{\prime}-W_{\Lambda}^{\prime} \sim \frac{1}{2 \phi}, \quad Z_{0}^{\prime}-Z_{\Lambda}^{\prime} \sim \frac{1}{12 \phi^{4}}, \quad X_{0}-X_{\Lambda} \sim \frac{1}{18 \phi^{6}}, \quad Y_{0}-Y_{\Lambda} \sim-\frac{1}{9 \phi^{7}} .
$$

As expected, the higher-order operators show a faster decay for large field values, see figure 3 and 4 .

\subsection{Supersymmetry breaking}

If we choose the classical superpotential to be a polynomial of the form $W_{\Lambda}^{\prime}(\phi) \sim \mathcal{O}\left(\phi^{n}\right)$ with leading power $n$ even, we expect spontaneous supersymmetry breaking to occur during the flow towards the IR $[6,17,31]$. It is known that spontaneous supersymmetry breaking is an IR effect, where the ground state is lifted to $E_{0}>0$ [32]. 


\subsubsection{Problems with the expansion in powers of $F$}

In order to study SUSY breaking within the FRG framework we focus on the $\mathbb{Z}_{2}$ symmetric even function

$$
W_{\Lambda}^{\prime}(\phi)=e+g \phi^{2}, \quad e<0, g>0 .
$$

Then the RG flow preserves the symmetry and $W_{k}^{\prime}(\phi)$ will remain $\mathbb{Z}_{2}$ symmetric for all scales.

For unbroken supersymmetry we employed an expansion in the auxiliary field around $F=0$ to derive the flow equations in terms of the scalar fields $\phi$. However, this expansion point is inappropriate when supersymmetry is broken in which case the vacuum expectation value of the average field $F$ does not vanish. The problem with expanding around $F=0$ can be seen already in the LPA where $W^{\prime \prime}(\phi)$ represents a "mass term" in the denominator of the flow equation. Hence, the regulator $r_{1}$ does not regulate since $\mathcal{W}^{\prime \prime}(\phi)=W^{\prime \prime}(\phi)+k$ will vanish for some value(s) of $\phi$. This means that the RG equation detects the massless fermionic excitation - the Goldstino mode - associated with the spontaneous breaking of supersymmetry. This mode mediates between the two degenerate ground states at $E_{0}>0$, one in the bosonic and one in the fermionic sector [6]. Hence, at the minimum of $V(\phi)$ the denominator in the flow equation simply contains the squared Goldstino mass $m_{G}^{2}=$ $W^{\prime \prime}(0)^{2}=0$. Thus, the flow of the superpotential diverges in the IR limit at the origin. This apparently leads to infinitely large excited energies, since $E_{1}=W^{\prime}(0) W^{(3)}(0)>0$ for broken supersymmetry. We find that this divergence occurs independently of the choice of the regulator $r_{2}$ and of the order of truncation. ${ }^{5}$ Thus, we are lead to Taylor-expand in powers of $F-F_{0}$ with non-vanishing $F_{0}$. We shall do this in NLO in the derivative expansion. First we consider the equation of motion for the auxiliary field in NLO, given by

$$
F=-i W_{k}^{\prime}(\phi) / Z_{k}^{\prime}(\phi)^{2} \text {. }
$$

If supersymmetry is spontaneously broken, $W_{k}^{\prime}(\phi)>0$ for all $\phi$. Again we observe that $F$ assumes a finite vacuum expectation value implying a breakdown of the flow equation when $W^{\prime}$ ceases to have a zero. Now we expand around a non-zero auxiliary field - determined by its equation of motion - and rewrite the l.h.s. of the flow equation as

$$
\begin{aligned}
\text { lhs } & =i F \partial_{k} W^{\prime}+\frac{1}{2}\left(\partial_{k} Z^{\prime 2}\right) F^{2}+\mathcal{O}\left(F^{3}\right) \\
& =\frac{W^{\prime}\left(Z^{\prime} \partial_{k} W^{\prime}-W^{\prime} \partial_{k} Z^{\prime}\right)}{Z^{\prime 3}}+i\left(\partial_{k} W^{\prime}-\frac{2 W^{\prime} \partial_{k} Z^{\prime}}{Z^{\prime}}\right)\left(F-F_{0}\right)+Z^{\prime} \partial_{k}\left(Z^{\prime}\right)\left(F-F_{0}\right)^{2}+\ldots \\
& \text { with } F_{0}(\phi)=-i W^{\prime}(\phi) / Z^{\prime}(\phi)^{2} .
\end{aligned}
$$

Obviously the term $\mathcal{O}\left(F^{3}\right)$ will contribute to all orders around this new expansion point. Unfortunately, there is no unique projection onto the flows of $W^{\prime}$ and $Z^{\prime}$. We may project onto the constant, the linear or the quadratic term in $\left(F-F_{0}\right)$. Hence, the system is overdetermined. Note that higher order terms contain no information about the flows of

\footnotetext{
${ }^{5}$ Of course, this IR problem represents a low dimensional issue as the divergences diminish with increasing dimension $d$, see $[16,17,23]$.
} 
$W^{\prime}$ and $Z^{\prime}$. Solving all three equations using an expansion of the r.h.s. of the flow equation yields no consistent solution, since higher derivative operators contribute to these lower orders as well. To obtain a maximally self-consistent truncation it is therefore necessary to minimize these contributions. Assuming a nice convergence behaviour of the derivative expansion, it is sensible to project onto the lowest orders in $\left(F-F_{0}\right)$.

\subsubsection{Numerical results}

In order to solve the flow equations for $W^{\prime}$ and $Z^{\prime}$ we now limit our discussion to the approximation of a uniform wave-function renormalization by setting $Z_{k}^{\prime}(\phi)=Z_{k}^{\prime}$. This corresponds to neglecting the field- and momentum dependence of $Z^{\prime}$. Then, the r.h.s. of the RG equation (2.12) simplifies to

$$
\begin{aligned}
\operatorname{rhs}= & \frac{1}{2} \int_{-k}^{k} \frac{\mathrm{dq}}{2 \pi}\left(\partial_{k}\left(Z^{\prime 2} r_{2}\right)\left(\mathcal{W}^{\prime \prime 2}-B^{2} q^{2}\right)-2\left(\partial_{k} r_{1}\right) B \mathcal{W}^{\prime \prime}\right)\left[\frac{W^{\prime} W^{\prime \prime \prime}}{\mathcal{N}\left(\mathcal{N} Z^{\prime 2}+B W^{\prime} W^{\prime \prime \prime}\right)}\left(F-F_{0}\right)^{0}\right. \\
& \left.+\frac{i W^{\prime \prime \prime} Z^{\prime 4}}{\left(\mathcal{N} Z^{\prime 2}+B W^{\prime} W^{\prime \prime \prime}\right)^{2}}\left(F-F_{0}\right)^{1}+\frac{B\left(W^{\prime \prime \prime}\right)^{2} Z^{\prime 6}}{\left(\mathcal{N} Z^{\prime 2}+B W^{\prime} W^{\prime \prime \prime}\right)^{3}}\left(F-F_{0}\right)^{2}+\ldots\right] \quad(2.41) \\
& \text { with } \mathcal{N}=B^{2} q^{2}+\mathcal{W}^{\prime \prime 2}, \quad B=Z^{\prime 2}\left(1+r_{2}\right), \quad \mathcal{W}^{\prime \prime}=W^{\prime \prime}+r_{1} .
\end{aligned}
$$

Note that we identified the wave-function renormalization $Z^{\prime}(\bar{\phi})$ which accompanies the regulator function $r_{2}$ with the field-independent $Z^{\prime}$. To analyze the flow of the effective average potential we proceed in two steps. First we start with a classical potential of the form (2.11) in the UV at $k=\Lambda$. Down to some scale $k_{0}>0, W^{\prime}$ will have a zero. In this regime $k \in\left(k_{0}, \Lambda\right)$ we employ the flow equations obtained by an expansion around $F=0$. Starting with $k_{0}$ down to the IR limit $k=0$ the scale-dependence of $W^{\prime}, Z^{\prime}$ is determined by the flow equations derived via an expansion around $F_{0} \neq 0$. As regulator functions we choose $r_{1}=0$ and $r_{2}=\left(k^{2} / p^{2}-1\right) \theta\left(k^{2}-p^{2}\right)$. To calculate the ground state energies $E_{0}$ we Taylor-expand $W^{\prime}$ about $\phi=0$ up to some order and solve the system of coupled ordinary differential equations numerically. This is a sensible approach when $W^{\prime}$ becomes flat in the IR, because due to supersymmetry the physics happens at vanishing field, in contrast to e.g. usual $O(N)$-models [24, 25], where the situation is exactly opposite: in the unbroken regime, the derivative of the potential is positive, whereas in the broken phase, one has a zero. As in the case of unbroken supersymmetry, we compare our results for $E_{0}$ with the ones obtained by numerically diagonalizing the Hamiltonian of the system.

Figure 5 displays the ground state energies as well as the relative error $e_{\text {trunc }}$ in LPA and NLO as obtained via two different projection methods. Here, $(i, j)$ corresponds to a projection onto $\left(F-F_{0}\right)^{i}$ and $\left(F-F_{0}\right)^{j}$. Apparently, the results are significantly improved by including a constant wave-function renormalization. In particular, this applies to large couplings $g$, where the relative error is approximately $4 \%$. Contrary to unbroken supersymmetry, the relative error increases with decreasing $g$. This originates from the fact that for decreasing $g$ the minima of the potential drift apart and tunneling effects become effective, see [20].

In NLO, a $(0,2)$-projection shows a smaller relative error than the (0,1)-projection up to some $g_{\text {max }} \approx 3.6$, since the flow of $Z^{\prime}$ slows down when including the higher order term 

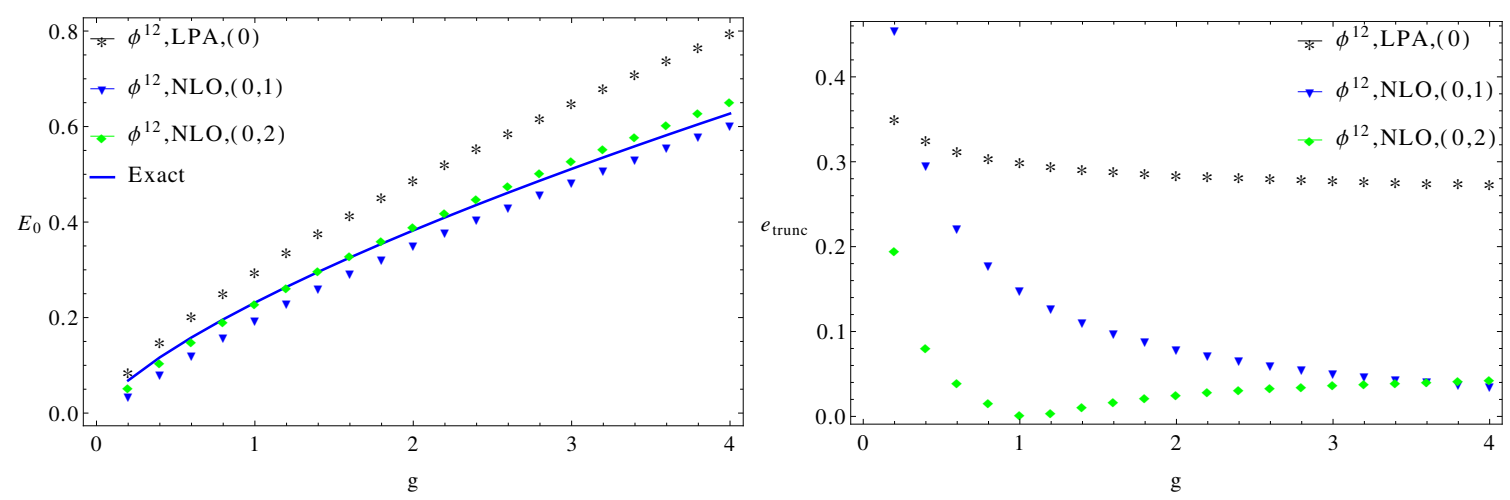

Figure 5. Ground state energy $E_{0}$ and its relative error $e_{\text {trunc }}$ for initial potentials of the form $W_{\Lambda}=$ $-0.1+\frac{g}{3} \phi^{3}$ as a function of $g$ obtained via a polynomial expansion of $W_{k}^{\prime}(\phi)$ up to $\phi^{12}$. The brackets encode the projection scheme, i.e. $(i, j)$ corresponds to a projection onto $\left(F-F_{0}\right)^{i}$ and $\left(F-F_{0}\right)^{j}$.

$\left(F-F_{0}\right)^{2}$ resulting in a higher ground state energy $E_{0}=V(0)=W^{\prime}(0) / Z^{\prime}(0)^{2}$. However, for large $g>g_{\max }$ the $(0,1)$-projection leads to superior results. This may be due to a larger truncation error in $\left(F-F_{0}\right)^{2}$ compared to $\left(F-F_{0}\right)^{1}$ with increasing coupling $g$, originating from the missing higher order terms $X, Y$ which are of importance there.

\section{$3 \mathcal{N}=1$ Wess-Zumino model in 3 dimensions}

As a second testing ground for the convergence properties of the supercovariant derivative expansion we choose the three-dimensional $\mathcal{N}=1$ Wess-Zumino model. This model has been examined in next-to-leading order in the derivative expansion with a momentum- and field-independent wave-function renormalization $Z_{k}$ in [17]. It was shown that at zero temperature, this model possesses an analogue of the Wilson-Fisher fixed point, separating the supersymmetric (spontaneously broken $\mathbb{Z}_{2}$ ) from the nonsupersymmetric $\left(\mathbb{Z}_{2}\right.$-symmetric) phase.

\subsection{Preliminaries}

Here, we shortly recall the main properties of the three-dimensional model. For more details in the context of flow equations we refer to [17]. The real scalar field $\phi$, real auxiliary field $F$ and real fermion field $\psi$ are components of a real superfield

$$
\Phi(x, \theta)=\phi(x)+\bar{\theta} \psi(x)+\frac{1}{2} \bar{\theta} \theta F(x) .
$$

The supersymmetry variations $\delta_{\epsilon} \Phi$ are generated by fermionic supercharges $\mathcal{Q}, \overline{\mathcal{Q}}$, where $\mathcal{Q}=-i \partial_{\bar{\theta}}-\not \partial \theta$ and $\overline{\mathcal{Q}}=-i \partial_{\theta}-\bar{\theta} \not \partial$ with anticommutation relations $\left\{\mathcal{Q}_{k}, \overline{\mathcal{Q}}_{l}\right\}=2 i \not \not_{k l}$. The supercovariant derivatives - fulfilling the corresponding relation $\left\{D_{k}, \bar{D}_{l}\right\}=-2 i \not \not_{k l}-$ read

$$
D=\partial_{\bar{\theta}}+i \not \partial \theta, \quad \text { and } \quad \bar{D}=-\partial_{\theta}-i \bar{\theta} \not \partial .
$$

Since there are no Majorana fermions in 3 Euclidean dimensions we switch to Minkowskispace with metric tensor $\eta_{\mu \nu}=\operatorname{diag}(1,-1,-1)$ and $\gamma$-matrices $\gamma^{\mu}=\left(\sigma_{2}, i \sigma_{3}, i \sigma_{1}\right)$, where 
$\mu=0,1,2$. After setting up the flow equation we return to Euclidean space, see [17]. With the above definitions we are able to construct the off-shell supersymmetric action in $\mathbb{R}^{1,2}$ superspace:

$$
S[\Phi]=\int d z\left[-\frac{1}{2} \Phi K \Phi+2 W(\Phi)\right], \quad K=\frac{1}{2}(\bar{D} D-D \bar{D}),
$$

where $z=(x, \theta, \bar{\theta})$ denotes the coordinates in superspace. After integration over the Majorana Grassmann variables $\theta, \bar{\theta}$ and elimination of the auxiliary field $F$ via its equation of motion $F=-W^{\prime}(\phi)$, we arrive at the following on-shell action in components,

$$
S_{\text {on }}[\phi, \psi, \bar{\psi}]=\int d^{3} x\left[\frac{1}{2} \partial_{\mu} \phi \partial^{\mu} \phi-\frac{i}{2} \bar{\psi} \not \partial \psi-\frac{1}{2} W^{\prime 2}(\phi)-\frac{1}{2} W^{\prime \prime}(\phi) \bar{\psi} \psi\right] .
$$

The last term in ( 3.4$)$ describes a Yukawa interaction between the scalars and fermions and $V(\phi)=W^{\prime 2}(\phi) / 2$ the potential self-energy of the scalars.

\subsection{Derivation of flow equation}

Now we use the flow equation in Minkowski spacetime

$$
\partial_{k} \Gamma_{k}=\frac{i}{2} \operatorname{STr}\left\{\partial_{k} R_{k}\left[\Gamma_{k}^{(2)}+R_{k}\right]^{-1}\right\}
$$

and perform a Wick rotation of the zeroth momentum component afterwards to obtain the corresponding flow in Euclidean space.

Analogously to eq. (2.15), the general ansatz for the scale-dependent effective average action reads

$$
\Gamma_{k}[\Phi]=\int \mathrm{d} z\left[2 W_{k}(\Phi)-\frac{1}{2} Z_{k}(\Phi) K Z_{k}(\Phi)-\frac{1}{8} Y_{1, k}(\Phi) K^{2} \Phi-\frac{1}{8} Y_{2, k}(\Phi)(K \Phi)(K \Phi)\right]
$$

with the scale- and field-dependent functions $W_{k}, Z_{k}, Y_{1, k}, Y_{2, k}$. We chose the prefactors of each term such that the resulting flow equations in Euclidean space exactly match the corresponding flow equations derived in supersymmetric quantum mechanics with $\int \frac{d q}{2 \pi} \rightarrow$ $\int \frac{d^{3} q}{(2 \pi)^{3}}$. By integrating over the anticommuting Grassmann variables in (3.6) we get

$$
\begin{aligned}
& \Gamma_{k}[\Phi]=\int d^{3} x\left[\frac{1}{2}\left(\partial_{\mu} Z\right)\left(\partial^{\mu} Z\right)-\frac{i}{2}\left(Z^{\prime} \bar{\psi}\right) \not \partial\left(Z^{\prime} \psi\right)-\frac{1}{4} Y_{1}^{\prime} \bar{\psi} \partial^{2} \psi-\left(\frac{1}{2} W^{\prime \prime}+\frac{1}{8} Y_{1}^{\prime \prime}\left(\partial^{2} \phi\right)\right) \bar{\psi} \psi\right. \\
& +\frac{1}{4} Y_{2}\left(\partial^{\mu} \bar{\psi}\right)\left(\partial_{\mu} \psi\right)+\left(W^{\prime}-\frac{1}{2} Z^{\prime} Z^{\prime \prime} \bar{\psi} \psi+\frac{1}{2}\left(Y_{1}^{\prime}+Y_{2}\right)\left(\partial^{2} \phi\right)+\frac{1}{4} Y_{1}^{\prime \prime}\left(\partial_{\mu} \phi\right)\left(\partial^{\mu} \phi\right)+\frac{i}{2} Y_{2}^{\prime} \bar{\psi} \not \partial \psi\right) F \\
& \left.+\left(\frac{1}{2} Z^{\prime 2}+\frac{1}{8} Y_{2}^{\prime \prime} \bar{\psi} \psi\right) F^{2}-\frac{1}{4} Y_{2}^{\prime} F^{3}\right]
\end{aligned}
$$

where again we ordered the terms in powers of the auxiliary field $F$. In analogy to (2.19), we assume the supersymmetric cutoff action to be of the form

$$
\Delta S_{k}[\Phi]=\frac{1}{2} \int \mathrm{d} z \Phi\left[2 r_{1}\left(-\partial_{\mu} \partial^{\mu}, k\right)-Z_{k}^{\prime 2}(\bar{\Phi}) r_{2}\left(-\partial_{\mu} \partial^{\mu}, k\right) K\right] \Phi,
$$


with $Z_{k}^{\prime}$ evaluated at the background field $\bar{\Phi}=\bar{\phi}$. As in quantum mechanics, we extract the scale dependence of $W^{\prime}, Z^{\prime}$ and $Y_{2}^{\prime}$ by projecting the r.h.s. of (3.5) onto $F, F^{2}$ and $F^{3}$ for constant bosonic fields and a vanishing fermion field. This way we obtain (cf. eq. (2.25))

$$
\begin{aligned}
& \left.\partial_{k} \Gamma_{k}\right|_{\partial_{\mu} \phi=\partial_{\mu} F=\psi=\bar{\psi}=0}=\int \mathrm{d}^{3} x\left(\partial_{k} W^{\prime} F+\frac{1}{2} \partial_{k} Z^{\prime 2} F^{2}-\frac{1}{4} \partial_{k} Y_{2}^{\prime} F^{3}\right) \\
& =\frac{i}{2} \int \frac{\mathrm{d}^{3} q}{(2 \pi)^{3}} \frac{\mathrm{d}^{3} q^{\prime}}{(2 \pi)^{3}} \mathrm{~d} \theta \mathrm{d} \bar{\theta} \mathrm{d} \theta^{\prime} \mathrm{d} \bar{\theta}^{\prime}\left(\partial_{k} R_{k}\right)\left(q^{\prime}, q, \theta^{\prime}, \theta\right) G_{k}\left(q, q^{\prime}, \theta, \theta^{\prime}\right) \\
& =\frac{i}{2} \int \mathrm{d}^{3} x \frac{\mathrm{d}^{3} q}{(2 \pi)^{3}}\left[\left(2 \partial_{k} r_{1}\right)(b+c+d)+\partial_{k}\left(r_{2} Z_{k}^{\prime 2}(\bar{\phi})\right)\left(-2 f q^{2}+a q^{2}+4 e\right)\right],
\end{aligned}
$$

where $(a, b, c, d, e, f)$ again represent the coefficients of the Greens function $G_{k}$, see appendix C. Finally, we obtain the flow equations in Euclidean space with metric $-\delta_{\mu \nu}$ via a Wick rotation of the zeroth momentum component, i.e. $q^{0} \rightarrow i q^{0}$. As mentioned above, these equations are by construction identically to the ones derived in SUSY quantum mechanics up to an integration over a three dimensional momentum space.

The missing flow of $Y_{1}^{\prime}+Y_{2}$ is derived in an exactly similar manner to $d=1$ as presented in appendix B by considering momentum-dependent fields $\phi, F$ and projecting onto the contribution in $\mathcal{Q}^{2} \delta \phi(\mathcal{Q}) \delta F(-\mathcal{Q})$ with an additional Wick rotation of $q^{0}$ afterwards. Finally, we define and substitute $Y:=Y_{2}^{\prime}$ and $X:=Y_{1}^{\prime}+Y_{2}$ in order to simplify the equations.

\subsection{Superscaling relation \& Wilson-Fisher fixed point}

In this section we study the fixed-point structure of the three-dimensional Wess-Zumino model in NNLO. To begin with, we have to choose suitable regulator functions. At the cutoff scale we assume $W_{\Lambda}^{\prime}$ to be an even polynomial. Then its leading power remains even during the flow and we can investigate the dynamical breaking of supersymmetry during the flow to the IR. The flow equations automatically force $W^{\prime}(\phi)$ to remain even for all scales $k<\Lambda$. Furthermore, the equations then imply that $Z^{\prime}(\phi)$ and $Y(\phi)$ are even and $X(\phi)$ is odd. If $W^{\prime}(\phi)$ tends asymptotically to an even power, $W^{\prime \prime}(\phi)$ always has a node which is merely shifted but not screened by the mass-like regulator $r_{1}$. Thus, we may set $r_{1}=0$. We select an optimized regulator function $r_{2}$ of the form ${ }^{6}$

$$
r_{2}\left(q^{2}, k\right)=\left(\frac{k^{n}}{|q|^{n}}-1\right) \Theta\left(\frac{k^{2}}{q^{2}}-1\right) \quad \text { with } n=2 .
$$

We identify the value of the background field appearing in the cutoff action with the minimum of the potential, i.e. $\bar{\phi}=\phi_{0}$. We emphasize that we do not stick to the background field approximation $[11,15]$ going along with the identification $\bar{\phi}=\phi$ for reasons given later in this section.

Next, we switch to dimensionless quantities in order to analyze the characteristics of the phase transition in $d=3$. With the canonical dimensions

$$
[\phi]=1 / 2, \quad[W]=2, \quad\left[Z^{\prime}\right]=0, \quad[X]=-1, \quad[Y]=-3 / 2,
$$

\footnotetext{
${ }^{6}$ The choice (3.10) with $n=1$ as used in [17] would lead to IR divergent flows of the forth-order operators $X$ and $Y$.
} 
we are lead to the transformations

$$
\begin{aligned}
& \chi=Z^{\prime}\left(\phi_{0}\right) k^{-1 / 2} \phi, \quad w(\chi)=W(\phi) k^{-2}, \quad z^{\prime}(\chi)=Z^{\prime}(\phi) / Z^{\prime}\left(\phi_{0}\right), \\
& x(\chi)=X(\phi) k / Z^{\prime 2}\left(\phi_{0}\right), \quad y(\chi)=Y(\phi) k^{3 / 2} / Z^{\prime 3}\left(\phi_{0}\right),
\end{aligned}
$$

with $\phi_{0}$ denoting the minimum of the potential. Employing the definition of the anomalous dimension

$$
\eta(k)=-\frac{d}{d t} \ln \left(Z^{\prime 2}\left(\phi_{0}\right)\right), \quad t=\ln (k / \Lambda),
$$

as well as the dimensionless momentum variable $u=q^{2} / k^{2}$, the flow of the dimensionless superpotential reads

$$
\begin{aligned}
& \partial_{t} w^{\prime}+\frac{1}{2}(3-\eta) w^{\prime}-\frac{1}{2}(\eta+1) \chi w^{\prime \prime}= \\
& =\frac{1}{2 \pi^{2}} \int_{0}^{1} \mathrm{~d} u \frac{\sqrt{u}(\eta(u-1)+2)\left(\alpha^{\prime}\left(u \alpha^{2}-4 \beta^{2}\right)+16 \alpha u z^{\prime} z^{\prime \prime} \beta\right)}{2\left(u \alpha^{2}+4 \beta^{2}\right)^{2}}
\end{aligned}
$$

where we abbreviated $\alpha:=u x+2 w^{\prime \prime}$ and $\beta=1+u\left(z^{\prime 2}-1\right)$. The l.h.s. of (3.14) includes the dimensional and anomalous scaling, whereas the r.h.s. encodes the interactions amongst the operators according to our ansatz of $\Gamma_{k}$. Note that of the fourth-order contributions only $x$ and not $y$ directly couples to the flow of the superpotential. The expressions of the remaining flows are rather long and therefore not written down explicitly.

Now we analyze the physics at the Wilson-Fisher fixed point, corresponding to

$$
\partial_{t} \mathcal{O}_{*}=0, \quad \text { with } \quad \mathcal{O}=\left(w^{\prime}, z^{\prime}, x, y\right) .
$$

For large fields $|\chi| \gg 1$ the r.h.s. of eq. (3.14), i.e. the nontrivial flow, vanishes as we generally expect $\left|w_{*}^{\prime \prime}\right|$ to be large for a $\mathbb{Z}_{2}$-symmetric system. This holds for the remaining flows as well. Thus, the fixed-point solution for large $\chi$ is fixed by the anomalous and canonical scaling, leading to the asymptotic behaviour

$$
w_{*}^{\prime}(\chi) \sim \chi^{\left(\frac{3-\eta}{\eta+1}\right)}, \quad z_{*}^{\prime}(\chi) \sim \chi^{-\left(\frac{\eta}{\eta+1}\right)}, \quad x_{*}(\chi) \sim \chi^{-2}, \quad y_{*}(\chi) \sim \chi^{-3} .
$$

Thus, for positive $\eta$, the higher order functions vanish for large fields. In [17], one nonGaussian IR-stable fixed point as the supersymmetric analogue of the Wilson-Fisher fixed point has been spotted. It possesses one IR unstable direction defined by $w_{*}^{\prime}(0)$ with critical exponent $\theta_{0}=1 / \nu_{\mathrm{w}}$ which can be related to the anomalous dimension $\eta$ via the superscaling relation $1 / \nu_{\mathrm{w}}=(3-\eta) / 2[17]$.

To begin with, we show that this interesting superscaling relation holds true to all orders in the supercovariant derivative expansion of $\Gamma_{k}$ and derive its form for arbitrary $d \geq 2$.

To show this, let us note that the only fixed point equation which depends explicitly on $w^{\prime}$ is the one for $w^{\prime}$ itself. $^{7}$ Thus, we consider small fluctuations around the fixed-point solution in $w^{\prime}$-direction, i.e. $w^{\prime}(t, \chi)=w_{*}^{\prime}(\chi)+\delta w^{\prime}(t, \chi)$ and possible higher order operators

\footnotetext{
${ }^{7}$ Note that in the subsequent derivation we assume $\eta$ to be independent of a scale-dependent $\phi_{0}$. We expect corrections due to the running of the VEV to be small, which is confirmed by our numerical studies.
} 


\begin{tabular}{|l|lllllll|}
\hline approximation & $w_{*}^{\prime}(0)$ & $\chi_{0}$ & $\eta$ & $\theta_{0}$ & $\theta_{1}$ & $\theta_{2}$ & $\theta_{3}$ \\
\hline LPA & -0.0420 & 0.147 & & $3 / 2$ & -0.702 & -3.800 & -7.747 \\
NLO & -0.0292 & 0.150 & 0.186 & 1.407 & -0.771 & -1.642 & -3.268 \\
NNLO & -0.0294 & 0.149 & 0.180 & 1.410 & -0.715 & -1.490 & -2.423 \\
\hline
\end{tabular}

Table 2. Value of the superpotential $w_{*}^{\prime}(\chi=0)$ at the origin, location of minimum $\chi_{0}$ of the fixed-point potential, anomalous dimension and leading and subleading critical exponents of the Wilson-Fisher fixed point for different orders in the supercovariant derivative expansion.

evaluated at the fixed point. By linearizing the flow (3.14) - generalized to $d$ dimensions ${ }^{8}$ - in $\delta w^{\prime}$ we arrive at the fluctuation equation

$$
\partial_{t} \delta w^{\prime}=\left(\frac{\eta-d}{2}+\mathcal{F}(\chi) \partial_{\chi}+\mathcal{G}(\chi) \partial_{\chi}^{2}\right) \delta w^{\prime}
$$

Here, $\mathcal{F}$ and $\mathcal{G}$ are functionals obtained from the linearization. The critical exponents then correspond to the negative eigenvalues of the operator on the r.h.s. Apparently, a constant variation is an eigenfunction to this operator with eigenvalue $(\eta-d) / 2$. Since the flow of all higher operators of $\Gamma_{k}$ remain independent of $w^{\prime}$ this is true to all orders. Hence, we have verified the superscaling relation

$$
\frac{1}{\nu_{\mathrm{w}}}=\frac{1}{2}(d-\eta), \quad d \geq 2
$$

Next we present the numerical results to the fixed point equations. We solved the fixed point equation globally via a combination of Chebyshev and rational Chebyshev polynomials. The fixed-point solutions of the four operators considered are illustrated in figure 6 . The IR relevant coupling $w^{\prime}(0)$, the location of the minimum of the potential as well as the anomalous dimension and leading critical exponents are displayed in table 2 . We observe a nice convergence behaviour with increasing order in the derivative expansion. This confirms that the quantitative relevance of the operators in $\Gamma_{k}$ correlates with their scaling dimension $D$ (canonical plus anomalous scaling). They are ordered as

$$
D_{w^{\prime}}>D_{z^{\prime}}>D_{x}>D_{y}
$$

and are determined in terms of $\eta$ as follows:

$$
D_{w^{\prime}}=\frac{3}{2}-\frac{\eta}{2}, \quad D_{z^{\prime}}=-\frac{\eta}{2}, \quad D_{x}=-(1+\eta), \quad D_{y}=-\frac{3}{2}(1+\eta) .
$$

Finally, we wish to comment on our decision not to use the background field approximation (BFA) for the spectrally adjusted regulator $r_{2}$. According to (3.16), the fixed point solution $z_{*}^{\prime}$ vanishes for large fields $|\chi| \rightarrow \infty$. Implementing the BFA goes along with the replacement $z^{\prime}\left(\chi_{0}\right)=1 \rightarrow z^{\prime}(\chi)$ in the dimensionless regulator. Thus, the regulator is suppressed artificially for large fields. This in turn can lead to instabilities. Indeed, during

\footnotetext{
${ }^{8}$ The r.h.s. of (3.14) holds for arbitrary $d$ up to a different dimensional prefactor of $1 /\left(2^{d-1} \pi^{d / 2} \Gamma(d / 2)\right)$.
} 

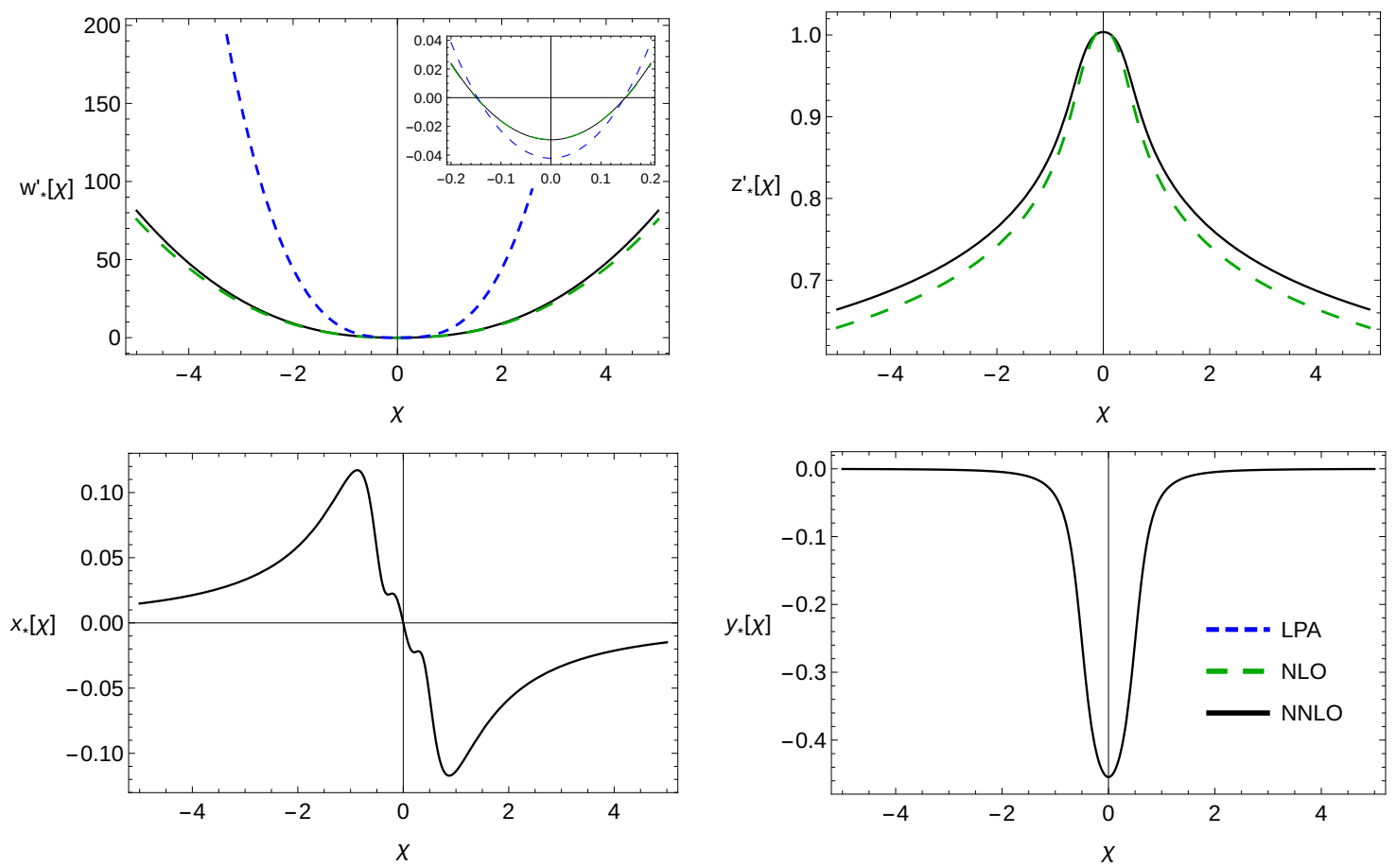

Figure 6. Fixed-point solutions of the three-dimensional Wess-Zumino model in LPA, NLO and NNLO. The change in asymptotics when going from LPA to NLO induced by the anomalous dimension is clearly visible. Also, the potential as well as the wave function renormalization change only mildly when going from NLO to NNLO, indicating once more a good convergence of the derivative expansion.

our numerical investigation of the fixed point equations we could not find a global solution in NNLO when employing the background field approximation, even though a solution via a Taylor expansion seems to exist. The difference between the physical quantities (critical exponents etc.) obtained by a Taylor expansion with BFA and the spectral method without BFA are almost identical. Thus one might argue that the error made in this approximation is irrelevant. However, one should bear in mind that a fixed point potential better be globally defined, and that there might be systems that are unstable against such types of approximations. This behaviour might also be explained via an analysis along the lines of [33], but this is beyond the scope of the present paper. Note also that when integrating the dimensionful flow equations, the difference should be even smaller as $Z^{\prime}(\phi)$ does not fall off asymptotically.

\section{Summary}

In this paper we studied the convergence of the derivative expansion in two supersymmetric theories via the functional renormalization group. Our approach includes a manifestly supersymmetric regulator and thus is suited to study systems with broken and unbroken supersymmetry. 
In the first part, we investigated supersymmetric quantum mechanical systems. Starting with symmetry-preserving flows, we obtained very good results for the energy gap within a truncation containing forth-order derivative terms. The relative error to the exact results is below one percent for a wide range of couplings $0<g<\sqrt{3}$ including the non-perturbative regime. For larger couplings, we observe a breakdown of the derivative expansion, which is expected since in this regime non-local instanton contributions play a crucial role. In the SUSY-breaking case, we studied the flow of the superpotential together with a constant wave-function renormalization. This required a suitable choice of projection to obtain the flow equations, because in the broken regime, the auxiliary field acquaints a finite expectation value which has to be accounted for. The results are again in agreement to exact results within a few percent.

The second part of this work deals with the Wess-Zumino model in 3 dimensions. In particular, we were interested in the analogue of the Wilson-Fisher fixed point of standard $O(N)$-theories. We were able to calculate all functionals at the fixed point up to forth order in supercovariant derivatives. Again, a very good convergence has been observed, and this convergence is further substantiated by two facts: on the one hand, the change of the fixed functionals is tiny when including forth-order terms in the effective average action. On the other hand, the critical exponents converge. Additionally, we could prove the superscaling relation to all orders in the derivative expansion.

In both studies, globally defined spectral methods were used to obtain the results. This ensures the numerical validity of our results, which are naturally free from any boundary effects usually present when applying a domain truncation.

\section{Acknowledgments}

The authors want to thank J. Borchardt, J.M. Pawlowski, R. Sondenheimer, F. SynatschkeCzerwonka and L. Zambelli for useful discussions. The research was supported by the Deutsche Forschungsgemeinschaft (DFG) graduate school GRK 1523/2. B. Knorr and A. Wipf thank the DFG for supporting this work under grant no. Wi777/11-1.

\section{A Greens function in $d=1$}

An analysis of the flow equation (2.12) requires the determination of the connected twopoint function $G_{k}$ as the inverse of $\left(\Gamma_{k}^{(2)}+R_{k}\right)$. In superspace, the relation $\mathbb{1}=G_{k}\left(\Gamma^{(2)}+R_{k}\right)$ is given by (2.23) with $G_{k}$ according to (2.24). The latter simply represents an expansion of $G_{k}$ in the Grassmann variables $\left(\theta, \theta^{\prime}\right)$ with arbitrary "bosonic" coefficients $(a, b, c, d, e, f)$. By solving (2.23), we finally obtain

$$
\begin{aligned}
& a=\frac{\left(B+\frac{3}{2} i F Y_{2}^{\prime}\right)}{\frac{1}{16}\left(4 A+F\left(3 F Y_{2}^{\prime \prime}-8 i Z^{\prime} Z^{\prime \prime}\right)\right)^{2}+\left(B+\frac{3}{2} i F Y_{2}^{\prime}\right) C} \\
& b=c=\frac{-\left(i A+\frac{3}{4} i F^{2} Y_{2}^{\prime \prime}+2 F Z^{\prime} Z^{\prime \prime}\right)}{\left(\frac{1}{16}\left(4 A+F\left(3 F Y_{2}^{\prime \prime}-8 i Z^{\prime} Z^{\prime \prime}\right)\right)^{2}+\left(B+\frac{3}{2} i F Y_{2}^{\prime}\right) C\right)} \\
& d=\frac{4 i}{4 A-4 i B q+F\left(F Y_{2}^{\prime \prime}+4 q Y_{2}^{\prime}-4 i Z^{\prime} Z^{\prime \prime}\right)}
\end{aligned}
$$




$$
\begin{aligned}
& e=\frac{4 i}{4 A+4 i B q+F\left(F Y_{2}^{\prime \prime}-4 q Y_{2}^{\prime}-4 i Z^{\prime} Z^{\prime \prime}\right)} \\
& f=\frac{1}{\left(B+\frac{3}{2} i F Y_{2}^{\prime}\right)}+\frac{\left(i A+\frac{3}{4} i F^{2} Y_{2}^{\prime \prime}+2 F Z^{\prime} Z^{\prime \prime}\right)^{2}}{\left(B+\frac{3}{2} i F Y_{2}^{\prime}\right)\left(\frac{1}{16}\left(4 A+3 F^{2} Y_{2}^{\prime \prime}-8 i F Z^{\prime} Z^{\prime \prime}\right)^{2}+\left(B+\frac{3}{2} i F Y_{2}^{\prime}\right) C\right)},
\end{aligned}
$$

where we introduced the abbreviations

$$
\begin{aligned}
A & =W^{\prime \prime}+r_{1}+\frac{1}{2}\left(Y_{1}^{\prime}+Y_{2}\right) q^{2} \\
B & =Z^{\prime 2}+r_{2} Z_{k}^{\prime 2}(\bar{\phi}) \\
C & =B q^{2}+\frac{i}{4} F^{3} Y_{2}^{\prime \prime \prime}+F^{2}\left(Z^{\prime \prime 2}+Z^{\prime \prime \prime} Z^{\prime}\right)+\frac{i}{2} F\left(q^{2}\left(2 Y_{2}^{\prime}+Y_{1}^{\prime \prime}\right)+2 W^{\prime \prime \prime}\right) .
\end{aligned}
$$

\section{B Flow equation of $Y_{1}^{\prime}+Y_{2}$}

This section explains the derivation of the flow of $Y_{1}^{\prime}+Y_{2}$. To derive the corresponding flow equation, we project the r.h.s. of (2.12) onto the time dependent term $\dot{F} \dot{\phi}$. This requires an expansion of the inverse propagator around field configurations of $F$ and $\phi$ exhibiting a small momentum dependence. Again, we therefore only consider the bosonic part of the superfield in the inverse propagator, i.e. we set $\psi$ and $\bar{\psi}$ equal to zero. The background field configurations in momentum space are then given by

$$
\begin{aligned}
\phi(p) & =\phi \delta(p)+\delta \phi(p)(\delta(p-Q)+\delta(p+Q)) \quad \text { and } \\
F(p) & =F \delta(p)+\delta F(p)(\delta(p-Q)+\delta(p+Q))
\end{aligned}
$$

with $\delta \phi(p) \ll \phi, \delta F(p) \ll F$. Note that $\phi(p)=\phi^{*}(-p)$ is real and $F(p)=-F^{*}(-p)$ purely imaginary. Next, we perform an expansion of the inverse Green's function $\Gamma_{k}^{(2)}+R_{k}$ up to $\mathcal{O}(\delta \phi(Q) \delta F(-Q))$ quadratic in the fluctuations. In the following, $z$ denotes the superspace coordinates $(q, \theta, \bar{\theta})$. Thus, the inverse propagator may be written in the form

$$
\left(\Gamma_{k}^{(2)}+R_{k}\right)\left(z, z^{\prime}\right)=\left[\Gamma_{0}\left(q, q^{\prime}\right)+\Gamma_{\phi}\left(q, q^{\prime}\right)+\Gamma_{F}\left(q, q^{\prime}\right)+\Gamma_{\phi F}\left(q, q^{\prime}\right)\right] \delta\left(\theta, \theta^{\prime}\right),
$$

corresponding to an expansion in powers of $\delta \phi$ and $\delta F$ with

$$
\begin{aligned}
\Gamma_{0}\left(q, q^{\prime}\right)= & \hat{\Gamma}_{0}(q) \delta\left(q-q^{\prime}\right) \quad \text { with } \\
& \hat{\Gamma}_{0}(q)=\left(i\left(W^{\prime \prime}+r_{1}\right)-B K(q)+\frac{i}{2}\left(Y_{1}^{\prime}+Y_{2}\right) q^{2}\right) \\
& \hat{\Gamma}_{0}^{-1}(q)=\frac{-i\left(W^{\prime \prime}+r_{1}+\frac{1}{2}\left(Y_{1}^{\prime}+Y_{2}\right) q^{2}\right)-B K(q)}{B^{2} q^{2}+\left(W^{\prime \prime}+r_{1}+\frac{1}{2}\left(Y_{1}^{\prime}+Y_{2}\right) q^{2}\right)^{2}} \\
\Gamma_{\phi}\left(q, q^{\prime}\right)= & \hat{\Gamma}_{\phi}(q, Q) \delta\left(q-q^{\prime}-Q\right)+\hat{\Gamma}_{\phi}(q,-Q) \delta\left(q-q^{\prime}+Q\right) \quad \text { with } \\
& \hat{\Gamma}_{\phi}(q, Q)=\left(i W^{\prime \prime \prime}+Z^{\prime} Z^{\prime \prime} Q^{2} \bar{\theta} \theta-Z^{\prime} Z^{\prime \prime}(K(q)+K(q-Q))-\frac{i}{2} Y_{2}^{\prime} Q^{2} \bar{\theta} \theta K(q-Q)\right. \\
& \left.-\frac{i}{2} Y_{2}^{\prime} Q^{2} K(q) \bar{\theta} \theta+\frac{i}{2} Y_{2}^{\prime} K(q) K(q-Q)+\frac{i}{4} Y_{1}^{\prime \prime}\left(q^{2}+Q^{2}+(q-Q)^{2}\right)\right) \delta \phi(Q), \\
\Gamma_{F}\left(q, q^{\prime}\right)= & \hat{\Gamma}_{F}(q, Q) \delta\left(q-q^{\prime}-Q\right)+\hat{\Gamma}_{F}(q,-Q) \delta\left(q-q^{\prime}+Q\right) \quad \text { with }
\end{aligned}
$$




$$
\begin{aligned}
& \hat{\Gamma}_{F}(q, Q)=\left(i W^{\prime \prime \prime} \bar{\theta} \theta+Z^{\prime} Z^{\prime \prime}-Z^{\prime} Z^{\prime \prime} K(q) \bar{\theta} \theta-Z^{\prime} Z^{\prime \prime} \bar{\theta} \theta K(q-Q)-\frac{i}{2} Y_{2}^{\prime} K(q)\right. \\
& \left.-\frac{i}{2} Y_{2}^{\prime} K(q-Q)+\frac{i}{2} Y_{2}^{\prime} K(q) \bar{\theta} \theta K(q-Q)+\frac{i}{4} Y_{1}^{\prime \prime}\left(q^{2}+Q^{2}+(q-Q)^{2}\right) \bar{\theta} \theta\right) \delta F(Q), \\
\Gamma_{\phi F}\left(q, q^{\prime}\right)= & \hat{\Gamma}_{\phi F}(q) \delta\left(q-q^{\prime}\right) \quad \text { with } \\
& \left.\hat{\Gamma}_{\phi F}(q)\right|_{\mathcal{O}\left(Q^{2}\right)}=\frac{i}{2}\left(Y_{2}^{\prime \prime}+Y_{1}^{\prime \prime \prime}\right) Q^{2} \bar{\theta} \theta(\delta \phi(Q) \delta F(-Q)+\delta F(Q) \delta \phi(-Q)) .
\end{aligned}
$$

Thereby, we considered only terms quadratic in $Q$ in $\Gamma_{\phi F}\left(q, q^{\prime}\right)$ as other terms do not contribute to the flow. Note that the operator $K$ occurring in eq. (B.3) is a function of the momentum as well as the Grassmann variables $\bar{\theta}, \theta$. Inserting the configuration (B.1) into (2.17) we obtain

$$
\frac{i}{2}\left(Y_{1}^{\prime}+Y_{2}\right)=\left.\frac{1}{\Omega} \lim _{Q^{2} \rightarrow 0} \frac{\partial}{\partial Q^{2}} \frac{\delta^{2} \Gamma_{k}}{\delta(\delta \phi(Q)) \delta(\delta F(-Q))}\right|_{\phi, F=\psi=\bar{\psi}=\delta F=\delta \phi=0},
$$

where $\Omega$ denotes the total volume of "space" and should be taken to infinity at the end. Now, the Green's function follows from (B.2) via an expansion in powers of $\delta \phi$ and $\delta F$. Keeping only contributions quadratic in the fluctuations $\sim(\delta F)(\delta \phi)$ we find

$$
\begin{aligned}
\left(G_{k}\right)\left(z, z^{\prime}\right)_{(\delta \phi \delta F)}= & \left\{-\hat{\Gamma}_{0}^{-1}(q) \Gamma_{\phi F}\left(q, q^{\prime}\right) \hat{\Gamma}_{0}^{-1}(q)\right. \\
& +\int_{\tilde{q}} \hat{\Gamma}_{0}^{-1}(q) \Gamma_{\phi}(q, \tilde{q}) \hat{\Gamma}_{0}^{-1}(\tilde{q}) \Gamma_{F}\left(\tilde{q}, q^{\prime}\right) \hat{\Gamma}_{0}^{-1}(q) \\
& \left.+\int_{\tilde{q}} \hat{\Gamma}_{0}^{-1}(q) \Gamma_{F}(q, \tilde{q}) \hat{\Gamma}_{0}^{-1}(\tilde{q}) \Gamma_{\phi}\left(\tilde{q}, q^{\prime}\right) \hat{\Gamma}_{0}^{-1}(q)\right\} \delta\left(\theta, \theta^{\prime}\right)
\end{aligned}
$$

Inserting the above propagator (B.5) into the flow equation and keeping only terms in $\delta \phi(Q) \delta F(-Q)$ we have

$$
\begin{aligned}
& \frac{i}{2} \partial_{k}\left(Y_{1}^{\prime}+Y_{2}\right) Q^{2} \delta \phi(Q) \delta F(-Q)=\frac{1}{2} \int \frac{\mathrm{d} q}{2 \pi} \mathrm{d} \theta \mathrm{d} \bar{\theta} \mathrm{d} \theta^{\prime} \mathrm{d} \bar{\theta}^{\prime} \partial_{k}\left[i r_{1}-Z_{k}^{\prime 2}(\bar{\phi}) r_{2} K\left(q, \theta^{\prime}\right)\right] \delta\left(\theta^{\prime}, \theta\right) \times \\
& \times\left[-\hat{\Gamma}_{0}^{-1}(q) \hat{\Gamma}_{\phi F}(q) \hat{\Gamma}_{0}^{-1}(q)+\hat{\Gamma}_{0}^{-1}(q) \hat{\Gamma}_{\phi}(q, Q) \hat{\Gamma}_{0}^{-1}(q-Q) \hat{\Gamma}_{F}(q-Q,-Q) \hat{\Gamma}_{0}^{-1}(q)\right. \\
& \left.\quad+\hat{\Gamma}_{0}^{-1}(q) \hat{\Gamma}_{F}(q,-Q) \hat{\Gamma}_{0}^{-1}(q+Q) \hat{\Gamma}_{\phi}(q+Q, Q) \hat{\Gamma}_{0}^{-1}(q)\right] \delta\left(\theta, \theta^{\prime}\right) .
\end{aligned}
$$

By integrating over the Grassmann variables and projecting the r.h.s. onto the contribution $\sim Q^{2}$ we thus get the flow of $Y_{1}^{\prime}+Y_{2}$.

\section{Greens function in $d=3$}

Similarly to the analysis in $d=1$, we determine the Greens function $G_{k}$ of the general form

$$
G_{k}\left(q, q^{\prime}, \theta, \theta^{\prime}\right)=\left(a+b \bar{\theta} \theta+c \bar{\theta}^{\prime} \theta^{\prime}+d \bar{\theta} \theta^{\prime}+e \bar{\theta} \theta \bar{\theta}^{\prime} \theta^{\prime}+f \bar{\theta}^{\prime} \not p \theta\right) \delta\left(q, q^{\prime}\right)
$$

by solving

$$
\int \frac{\mathrm{d}^{3} q^{\prime}}{(2 \pi)^{3}} \mathrm{~d} \theta^{\prime} \mathrm{d} \bar{\theta}^{\prime} G_{k}^{-1}\left(q, q^{\prime}, \theta, \theta^{\prime}\right) G_{k}\left(q^{\prime}, q^{\prime \prime}, \theta^{\prime}, \theta^{\prime \prime}\right)=\delta\left(q, q^{\prime \prime}\right) \delta\left(\theta, \theta^{\prime \prime}\right)
$$


for the coefficients $(a, b, c, d, e, f)$. This yields

$$
\begin{aligned}
& a=\frac{8\left(2 B-3 F Y_{2}^{\prime}\right)}{8 C\left(2 B-3 F Y_{2}^{\prime}\right)-\left(4 A-3 F^{2} Y_{2}^{\prime \prime}+8 F Z^{\prime} Z^{\prime \prime}\right)^{2}} \\
& b=c=\frac{8 A-6 F^{2} Y_{2}^{\prime \prime}+16 F Z^{\prime} Z^{\prime \prime}}{\left(4 A-3 F^{2} Y_{2}^{\prime \prime}+8 F Z^{\prime} Z^{\prime \prime}\right)^{2}+8 C\left(3 F Y_{2}^{\prime}-2 B\right)} \\
& d=-\frac{4\left(A-\frac{1}{4} F^{2} Y_{2}^{\prime \prime}+F Z^{\prime} Z^{\prime \prime}\right)}{4\left(A-\frac{1}{4} F^{2} Y_{2}^{\prime \prime}+F Z^{\prime} Z^{\prime \prime}\right)^{2}-4 q^{2}\left(B-F Y_{2}^{\prime}\right)^{2}} \\
& e=\frac{4 C}{8 C\left(2 B-3 F Y_{2}^{\prime}\right)-\left(4 A-3 F^{2} Y_{2}^{\prime \prime}+8 F Z^{\prime} Z^{\prime \prime}\right)^{2}} \\
& f=-\frac{16\left(B-F Y_{2}^{\prime}\right)}{\left(4 A-4 B q+F\left(-F Y_{2}^{\prime \prime}+4 q Y_{2}^{\prime}+4 Z^{\prime} Z^{\prime \prime}\right)\right)\left(4 A+4 B q-F\left(F Y_{2}^{\prime \prime}+4 q Y_{2}^{\prime}-4 Z^{\prime} Z^{\prime \prime}\right)\right)},
\end{aligned}
$$

where we have used the abbreviations

$$
\begin{aligned}
A & =W^{\prime \prime}+r_{1}-\frac{1}{2}\left(Y_{1}^{\prime}+Y_{2}\right) q^{2} \\
B & =Z^{\prime 2}+r_{2} Z_{k}^{\prime 2}(\bar{\phi}) \\
C & =B q^{2}-\frac{1}{4} F^{3} Y_{2}^{\prime \prime \prime}+F^{2}\left(Z^{\prime \prime 2}+Z^{\prime \prime \prime} Z^{\prime}\right)+\frac{1}{2} F\left(2 W^{\prime \prime \prime}-q^{2}\left(2 Y_{2}^{\prime}+Y_{1}^{\prime \prime}\right)\right) .
\end{aligned}
$$

\section{D (Pseudo-)spectral methods}

We obtained the numerical results in this work in part with so-called (pseudo-)spectral methods. Spectral methods were studied from a mathematical point of view already some decades ago, however, they were only applied in certain fields of physics up to now, e.g. in numerical relativity, meteorology or fluid mechanics. The basic idea behind spectral methods is to expand the solution into orthogonal polynomials which should be chosen to fit the problem. A well-known example is the Fourier series of a periodic function. In our case, we chose a combination of Chebyshev and rational Chebyshev polynomials in order to resolve the operators globally. On the other hand, in RG-time-direction, we chose to map the (infinite) time axis onto a finite interval, then slicing it into smaller pieces and apply a Chebyshev spectralization in this direction. With a stabilized Newton-Raphson iteration scheme, we demanded that the flow equations are satisfied on collocation points up to a certain tolerance. This twofold application of spectral methods was considered too expensive in former times, but thanks to the progress in computing power, it is feasible now. This point is also undermined by the recent application of this method to gain exact solutions to the Einstein field equations for axisymmetric and stationary space times [27, 34].

The reason to use spectral methods is their extraordinary speed of convergence. For well-behaved functions, a spectral method may convergence exponentially, i.e. faster than any power law. Another advantage is that the expansion coefficients give a rough estimate of the maximal error in the interpolation of the solution. A general rule of thumb is that the error is bounded by roughly the absolute value of the last coefficient retained. For an extensive review of spectral methods, see e.g. [26]. The application of pseudo-spectral methods to flow equations will be discussed in detail elsewhere [35]. 
Open Access. This article is distributed under the terms of the Creative Commons Attribution License (CC-BY 4.0), which permits any use, distribution and reproduction in any medium, provided the original author(s) and source are credited.

\section{References}

[1] A. Feo, Predictions and recent results in SUSY on the lattice, Mod. Phys. Lett. A 19 (2004) 2387 [hep-lat/0410012] [INSPIRE].

[2] J. Giedt, Deconstruction and other approaches to supersymmetric lattice field theories, Int. J. Mod. Phys. A 21 (2006) 3039 [hep-lat/0602007] [INSPIRE].

[3] S. Catterall, From twisted supersymmetry to orbifold lattices, JHEP 01 (2008) 048 [arXiv:0712.2532] [INSPIRE].

[4] G. Bergner, T. Kaestner, S. Uhlmann and A. Wipf, Low-dimensional supersymmetric lattice models, Annals Phys. 323 (2008) 946 [arXiv:0705.2212] [InSPIRE].

[5] T. Takimi, Relationship between various supersymmetric lattice models, JHEP 07 (2007) 010 [arXiv:0705.3831] [INSPIRE].

[6] C. Wozar and A. Wipf, Supersymmetry breaking in low dimensional models, Annals Phys. 327 (2012) 774 [arXiv:1107.3324] [INSPIRE].

[7] D. Baumgartner and U. Wenger, Exact results for supersymmetric quantum mechanics on the lattice, PoS(LATTICE 2011) 239 [arXiv:1201.1485] [INSPIRE].

[8] G. Bergner, I. Montvay, G. Münster, D. Sandbrink and U.D. Özugurel, $N=1$ supersymmetric Yang-Mills theory on the lattice, PoS (LATTICE 2013) 483 [arXiv:1311.1681] [INSPIRE].

[9] C. Wetterich, Exact evolution equation for the effective potential, Phys. Lett. B 301 (1993) 90 [INSPIRE].

[10] N. Tetradis and C. Wetterich, Critical exponents from effective average action, Nucl. Phys. B 422 (1994) 541 [hep-ph/9308214] [INSPIRE].

[11] M. Reuter and C. Wetterich, Effective average action for gauge theories and exact evolution equations, Nucl. Phys. B 417 (1994) 181 [INSPIRE].

[12] H. Gies and L. Janssen, UV fixed-point structure of the three-dimensional Thirring model, Phys. Rev. D 82 (2010) 085018 [arXiv: 1006.3747] [INSPIRE].

[13] T.K. Herbst, M. Mitter, J.M. Pawlowski, B.-J. Schaefer and R. Stiele, Thermodynamics of QCD at vanishing density, Phys. Lett. B 731 (2014) 248 [arXiv:1308.3621] [INSPIRE].

[14] N. Christiansen, B. Knorr, J.M. Pawlowski and A. Rodigast, Global flows in quantum gravity, arXiv:1403.1232 [INSPIRE].

[15] F. Synatschke, G. Bergner, H. Gies and A. Wipf, Flow equation for supersymmetric quantum mechanics, JHEP 03 (2009) 028 [arXiv:0809.4396] [INSPIRE].

[16] F. Synatschke, H. Gies and A. Wipf, Phase diagram and fixed-point structure of two dimensional $N=1$ Wess-Zumino models, Phys. Rev. D 80 (2009) 085007 [arXiv: 0907.4229] [INSPIRE].

[17] F. Synatschke, J. Braun and A. Wipf, $N=1$ Wess-Zumino model in $D=3$ at zero and finite temperature, Phys. Rev. D 81 (2010) 125001 [arXiv:1001.2399] [INSPIRE]. 
[18] A. Horikoshi, K.-I. Aoki, M.-A. Taniguchi and H. Terao, Nonperturbative renormalization group and quantum tunneling, hep-th/9812050 [INSPIRE].

[19] A.S. Kapoyannis and N. Tetradis, Quantum mechanical tunneling and the renormalization group, Phys. Lett. A 276 (2000) 225 [hep-th/0010180] [INSPIRE].

[20] D. Zappala, Improving the renormalization group approach to the quantum mechanical double well potential, Phys. Lett. A 290 (2001) 35 [quant-ph/0108019] [INSPIRE].

[21] H. Gies, Introduction to the functional RG and applications to gauge theories, Lect. Notes Phys. 852 (2012) 287 [hep-ph/0611146] [INSPIRE].

[22] A. Wipf, Statistical approach to quantum field theory, Lect. Notes Phys. 864 (2013) 1 [INSPIRE].

[23] F. Synatschke, H. Gies and A. Wipf, The phase diagram for Wess-Zumino models, AIP Conf. Proc. 1200 (2010) 1097 [arXiv:0909.4189] [InSPIRE].

[24] M. Heilmann, D.F. Litim, F. Synatschke-Czerwonka and A. Wipf, Phases of supersymmetric $O(N)$ theories, Phys. Rev. D 86 (2012) 105006 [arXiv:1208.5389] [INSPIRE].

[25] D.F. Litim, M.C. Mastaler, F. Synatschke-Czerwonka and A. Wipf, Critical behavior of supersymmetric $O(N)$ models in the large- $N$ limit, Phys. Rev. D 84 (2011) 125009 [arXiv:1107.3011] [INSPIRE].

[26] J.P. Boyd, Chebyshev and Fourier spectral methods, $2^{\text {nd }}$ ed., Dover Publications, New York U.S.A. (2000).

[27] M. Ansorg, A. Kleinwachter and R. Meinel, Highly accurate calculation of rotating neutron stars: detailed description of the numerical methods, Astron. Astrophys. 405 (2003) 711 [astro-ph/0301173] [INSPIRE].

[28] H. Gies, F. Synatschke and A. Wipf, Supersymmetry breaking as a quantum phase transition, Phys. Rev. D 80 (2009) 101701 [arXiv:0906.5492] [INSPIRE].

[29] A. Salam and J.A. Strathdee, Supergauge transformations, Nucl. Phys. B 76 (1974) 477 [INSPIRE].

[30] D.F. Litim, Optimized renormalization group flows, Phys. Rev. D 64 (2001) 105007 [hep-th/0103195] [INSPIRE].

[31] E. Witten, Dynamical breaking of supersymmetry, Nucl. Phys. B 188 (1981) 513 [InSPIRE].

[32] M. Dine and J.D. Mason, Supersymmetry and its dynamical breaking, Rept. Prog. Phys. $\mathbf{7 4}$ (2011) 056201 [arXiv: 1012. 2836] [INSPIRE].

[33] I.H. Bridle, J.A. Dietz and T.R. Morris, The local potential approximation in the background field formalism, JHEP 03 (2014) 093 [arXiv:1312.2846] [INSPIRE].

[34] R.P. Macedo and M. Ansorg, Axisymmetric fully spectral code for hyperbolic equations, J. Comput. Phys. 276 (2014) 357 [arXiv: 1402 .7343] [INSPIRE].

[35] J. Borchardt and B. Knorr, in preparation (2015). 